\title{
Update on role of agalsidase alfa in management of Fabry disease
}

\author{
This article was published in the following Dove Press journal: \\ Drug Design, Development and Therapy \\ 14 March 20II \\ Number of times this article has been viewed
}

\section{Uma Ramaswami \\ Paediatric Metabolic Unit, Cambridge University Hospitals, Cambridge, UK}

Correspondence: Uma Ramaswami Paediatric Metabolic Unit, Box I8I, Cambridge University Hospitals, Cambridge CB2 0QQ, UK

$\mathrm{Tel}+44$ I22 32I 6878

Fax +44 I22 3586794

Email uma.ramaswami@addenbrookes. nhs.uk
Abstract: Fabry disease (FD) is an X-linked lysosomal storage disorder that affects both men and women. The manifestations of this heterogeneous disease are multisystemic and progressive. Prior to the development of enzyme replacement therapy, the management and treatment for Fabry disease was largely nonspecific and supportive. Because enzyme replacement therapy became commercially available in 2001, a variety of clinical benefits in Fabry patients have been consistently reported, including improved renal pathology and cardiac function, and reduced severity of neuropathic pain and improved pain-related quality of life. This update focuses on published data on the efficacy and tolerability of enzyme replacement therapy with agalsidase alfa, and gives a brief overview on some of the outstanding management issues in the treatment of this complex disease.

Keywords: enzyme replacement therapy, Fabry disease, agalsidase alfa

\section{Introduction}

Anderson-Fabry disease (FD; OMIM 301500) is a metabolic storage disorder resulting from deficient activity of the lysosomal hydrolase, $\alpha$-galactosidase A ( $\alpha$-gal A). ${ }^{1}$ In individuals with defective $\alpha$-gal A, the lipid substrate globotriaosylceramide $(\mathrm{Gb} 3)$ accumulates in lysosomes throughout the body, resulting in progressive multiple-organ pathology. ${ }^{1}$ Early signs and symptoms characteristic of the enzymatic defect include recurrent episodes of acroparesthesia and neuropathic pain crises, angiokeratomas, hearing loss and tinnitus, hypohidrosis, and corneal lesions. ${ }^{2}$ With accumulating Gb3 deposits in lysosomes and increasing age, premature mortality usually occurs due to renal, cardiac, and/or cerebral complications. ${ }^{3}$ Fabry disease is a rare X-linked genetic trait affecting primarily males in all ethnic groups. Affected hemizygous males who have no or minimal $\alpha$-gal A activity exhibit the classic phenotype, with clinical manifestations presenting in childhood or adolescence. ${ }^{4}$ Most heterozygous females are affected, although $\alpha$-gal A activity level and the onset and rate of disease progression is more variable than for hemizygous males. ${ }^{5,6}$ In both heterozygous females and hemizygous males, lifespan is typically reduced by approximately 15-20 years. ${ }^{3,5}$ Classical Fabry disease, first described independently in 1898 by Fabry in Germany ${ }^{7}$ and by Anderson in England, ${ }^{8}$ is a rare disorder, with an estimated incidence of 1 per 117,000 live births. ${ }^{4,9}$ The clinical spectrum of classic Fabry disease has expanded in the past 15 years due to the discovery of milder phenotypes with residual $\alpha$-gal A activity, resulting in later-onset cardiac and renal manifestations. ${ }^{4,10-13} \mathrm{~A}$ recent Italian newborn screening study for Fabry disease determined the $\alpha$-gal A activity in dried blood spots of 37,104 consecutive newborn males. ${ }^{4}$ In this population, Spada et al reported the 
incidence of $\alpha$-gal A deficiency to be 15-20 times higher than previous estimates. ${ }^{4}$ Moreover, the higher incidence found by newborn screening of later-onset phenotypes compared with classic phenotypes (11:1 ratio of later-onset versus classic phenotypes, respectively) suggests Fabry disease is currently underrecognized. ${ }^{4}$ Similarly, a newborn screening study by Hwu et al screened 90,288 male Taiwanese newborns and reported a high incidence of approximately one in 1250 with Fabry disease mutations, $86 \%$ of which were mutations found in later-onset cardiac phenotype patients. ${ }^{14}$

Due to its rarity, the broad number and severity of symptoms, and the heterogeneous phenotypic expressions, diagnosis is often challenging, particularly for heterozygous females and later-onset phenotypes. ${ }^{4}$ Future newborn screening for Fabry disease, as well as screening of high-risk populations, such as male kidney recipients, could be implemented into existing screening programs to aid early diagnosis. ${ }^{15}$

Overall, clinical studies in adult Fabry patients suggest that early initiation of enzyme replacement therapy may be able to alleviate symptoms and slow disease progression. Enzyme replacement therapy appears to normalize Gb3 levels in various organs, with numerous reported symptomatic benefits. ${ }^{16-19}$ The Mainz Severity Score Index, which provides an overall measure of disease severity, shows a marked reduction of Fabry disease severity following at least 1 year of enzyme replacement therapy $(P<0.001) .{ }^{17}$ Fabry disease is a progressive disorder, so enzyme replacement therapy intervention in childhood has the potential to provide greater long-term benefits, such as reducing or eliminating major organ damage in later life. ${ }^{2,20,21}$ More recent studies evaluating long-term (up to 4 years of follow-up) enzyme replacement therapy in children with Fabry disease demonstrate that agalsidase alfa is well tolerated, with efficacy profiles consistent with those reported in adults. ${ }^{2,20,22}$ However, long-term follow-up studies are required to confirm that initiation of enzyme replacement therapy for Fabry disease during childhood can prevent the irreversible, life-threatening organ damage that can occur during adulthood.

\section{Availability of enzyme replacement therapy}

Two distinct recombinant protein replacement drugs are approved for use in Europe for the treatment of Fabry patients, ie, agalsidase alfa $\left(\right.$ Replagal $^{\circledR}$; Shire Human Genetic Therapies, Dublin, Ireland) and agalsidase beta (Fabrazyme ${ }^{\circledR}$; Genzyme Corporation, Cambridge, MA). Studies have shown that the two recombinant enzymes exhibit identical biochemical properties and are comparable with respect to amino acid composition, specific activity, stability, and uptake by cultured fibroblasts, with only minor differences in glycosylation composition and mannose-6-phosphate receptor-mediated cellular uptake. ${ }^{23-25}$ Both agalsidase alfa and agalsidase beta contain recombinant human $\alpha$-gal $\mathrm{A}$, but they are produced differently and are approved for administration at different doses (administered as an intravenous infusion every other week at $0.2 \mathrm{mg} / \mathrm{kg}$ for agalsidase alfa over 40 minutes $^{26}$ or $1.0 \mathrm{mg} / \mathrm{kg}$ for agalsidase beta over $1.5-4.5$ hours). ${ }^{18,27}$ As with other recombinant therapies, human $\alpha$-gal A treatment is expensive, and at the registered dose, the annual cost of both drugs is equal at approximately $€ 210,000$ per $70 \mathrm{~kg}$ patient. ${ }^{28}$ Agalsidase alfa is produced using cultured human skin fibroblasts with an activated promoter of the $\alpha$-gal A gene, and agalsidase beta is produced by classical transduction of Chinese hamster ovary cells with human $\alpha$-gal A cDNA. ${ }^{29,30}$

In June 2009, Genzyme reported viral contamination in the manufacturing process of Fabrazyme, which has led to a continuous global shortage of agalsidase beta. ${ }^{31}$ Updated treatment recommendations advising reduced dosing regimens have consequently been published by the European Medicines Agency for adult male patients currently receiving Fabrazyme. ${ }^{31,32}$ Switching to agalsidase alfa (Replagal) has also been initiated for some Fabry patients, with careful monitoring. Agalsidase alfa is licensed in Europe, as well as in Canada, Japan, New Zealand, and several countries in South America. ${ }^{33}$ It is currently an investigational product in the US. The impact of a Fabrazyme shortage and switching to Replagal with respect to the clinical outcome is currently unknown, and hence will not be discussed further. However, clinicians treating patients with Fabry disease continue to be in discussion with the European Medicines Agency to ensure all individual patients receive the best possible treatment option based on their clinical need.

\section{Pharmacology of agalsidase alfa}

Few studies have evaluated the pharmacokinetics and pharmacodynamics of agalsidase alfa. ${ }^{29} \mathrm{~A}$ single intravenous dose of agalsidase alfa $0.2 \mathrm{mg} / \mathrm{kg}$ has been shown to exhibit a biphasic serum distribution and elimination profile from the circulation in both adults and children. ${ }^{21,29,34}$ The pharmacokinetic properties of agalsidase alfa are essentially unaffected by the dose of the enzyme, and were not significantly different between male and female patients. ${ }^{29}$ Elimination half-lives were $108 \pm 17$ minutes in males compared with $89 \pm 28$ minutes in females, and volume of distribution was approximately $17 \%$ body weight in both 
genders. ${ }^{29}$ Clearance normalized for body weight was 2.66 and $2.10 \mathrm{~mL} / \mathrm{min} / \mathrm{kg}$ for males and females, respectively. ${ }^{29}$ A short plasma half-life, $<1 \%$ of the maximal plasma concentration of agalsidase alfa, detectable 8 hours after dosing, has also been reported. ${ }^{29,35,36}$ The intracellular half-life of agalsidase alfa is reported to be 24-48 hours. ${ }^{19}$

Following six months of agalsidase alfa treatment, 12 of 28 male patients showed altered pharmacokinetics, including an apparent increase in clearance. These changes were associated with the development of low titer antibodies to agalsidase alfa, but no clinically significant effects on safety or efficacy were observed in the patients studied. ${ }^{29}$ In children aged 7-18 years, agalsidase alfa $0.2 \mathrm{mg} / \mathrm{kg}$ is cleared faster from the circulation than in adult patients with Fabry disease $(P<0.0001) .{ }^{21,29}$

\section{Clinical efficacy in Fabry disease}

The evidence of clinical efficacy of agalsidase alfa in Fabry disease has been reported in 5 randomized clinical trials, 13 open-label studies, and 11 reports from the Fabry Outcome Survey. The Fabry Outcome Survey is one of the most comprehensive global observational database on patients with Fabry disease. Initiated and sponsored by Shire Human Genetic Therapies in 2001, the Fabry Outcome Survey aims to increase our understanding of the nature of Fabry disease and to improve the clinical management of all Fabry patients, including women and children, receiving enzyme replacement therapy with agalsidase alfa. As of September 2010, the Fabry Outcome Survey contains data on 1933 patients from 22 countries (Shire Human Genetic Therapies, data on file), and the inclusion criteria have been previously described. ${ }^{37}$

Table 1 summarizes these studies according to their primary endpoints. Plasma and urinary Gb3 levels, renal endpoints (including glomerular filtration rate), and cardiac and neurological endpoints were most commonly studied. The most recent and relevant studies are discussed relating to clinical outcome in this section.

\section{Effects on renal function}

Renal dysfunction is a major complication of Fabry disease, and end-stage renal disease is most common in affected males before the fourth decade. ${ }^{3,38,39} \mathrm{~A}$ smaller number of heterozygous females also have a poor renal prognosis. ${ }^{5} \mathrm{~Gb} 3$ gradually accumulates in lysosomes in renal cells, leading to microvascular dysfunction, occlusion, and ischemia, with subsequent development of tubular atrophy, segmental and global sclerosis, and interstitial fibrosis. ${ }^{38-41}$ Overall, long-term enzyme replacement therapy with agalsidase alfa shows stabilization of kidney function in patients with early stages of chronic kidney disease at baseline and slowing of progression of renal function compared with historical control subjects, even in male and female patients with advanced chronic kidney disease. ${ }^{26,42-44}$

Agalsidase alfa $0.2 \mathrm{mg} / \mathrm{kg}$ every other week was initially evaluated in a randomized, double-blind, placebo-controlled clinical trial of 26 adult males with Fabry disease. ${ }^{26}$ Inulin clearance and creatinine clearance were used to measure glomerular filtration rate at baseline and at week $24 .^{26}$ Following an initial six months of treatment with agalsidase alfa, creatinine clearance increased slightly compared with placebo, from $92.7 \pm 6.2 \mathrm{~mL} / \mathrm{min} / 1.73 \mathrm{~m}^{2}$ at baseline to $94.8 \pm 7.7 \mathrm{~mL} / \mathrm{min} / 1.73 \mathrm{~m}^{2}(P=0.02) .{ }^{26}$ Similar results were observed for glomerular filtration rate estimated by inulin clearance, although the results were not statistically significant. ${ }^{26}$ During a long-term, open-label extension of this study, glomerular filtration rate was estimated using the four-variable Modification of Diet in Renal Disease method using serial measurements of serum creatinine. ${ }^{42,45}$ The mean ( \pm standard deviation) estimated glomerular filtration rate of patients with Stage I disease (mean baseline estimated glomerular filtration rate $108.7 \pm 14.1 \mathrm{~mL} / \mathrm{min} / 1.73 \mathrm{~m}^{2}, \mathrm{n}=12$ ) or Stage II disease (mean baseline estimated glomerular filtration rate $78.6 \pm 8.2 \mathrm{~mL} / \mathrm{min} / 1.73 \mathrm{~m}^{2}, \mathrm{n}=8$ ) remained relatively stable after 4 years of treatment (mean estimated glomerular filtration rate $101.5 \pm 12.4 \mathrm{~mL} / \mathrm{min} / 1.73 \mathrm{~m}^{2}$ and $67.1 \pm 17.0 \mathrm{~mL} / \mathrm{min} / 1.73 \mathrm{~m}^{2}$, for patients with baseline Stage I and II disease, respectively). ${ }^{42}$ During this study, a subgroup of patients $(n=4)$ with more severe kidney dysfunction (mean baseline estimated glomerular filtration rate $47.1 \pm 9.4 \mathrm{~mL} / \mathrm{min} / 1.73 \mathrm{~m}^{2}$ ) showed a decrease to $25.1 \pm 16.4 \mathrm{~mL} / \mathrm{min} / 1.73 \mathrm{~m}^{2}, P=0.098$ ) following 48 months of enzyme replacement therapy; representing an average rate of decline of approximately $5.2 \mathrm{~mL} / \mathrm{min} / 1.73 \mathrm{~m}^{2}$. None of the patients with Stage III disease at baseline progressed to end-stage renal failure during this study. More recently, Schiffmann et al demonstrated that after switching to a weekly infusion of agalsidase alfa $0.2 \mathrm{mg} / \mathrm{kg}$ despite 2-4 years of conventional treatment of agalsidase alfa $0.2 \mathrm{mg} / \mathrm{kg}$ every 2 weeks, 9 of 11 patients $(82 \%)$ showed either an improvement in estimated glomerular filtration rate $(n=3)$ or a slowing in the rate of estimated glomerular filtration rate decline $(n=6)$; estimated glomerular filtration rate was calculated using the four-variable Modification of Diet in Renal Disease formula. ${ }^{46}$ During the 24-month follow-up period on weekly dosing, the mean rate of change in estimated glomerular filtration rate was observed to slow 


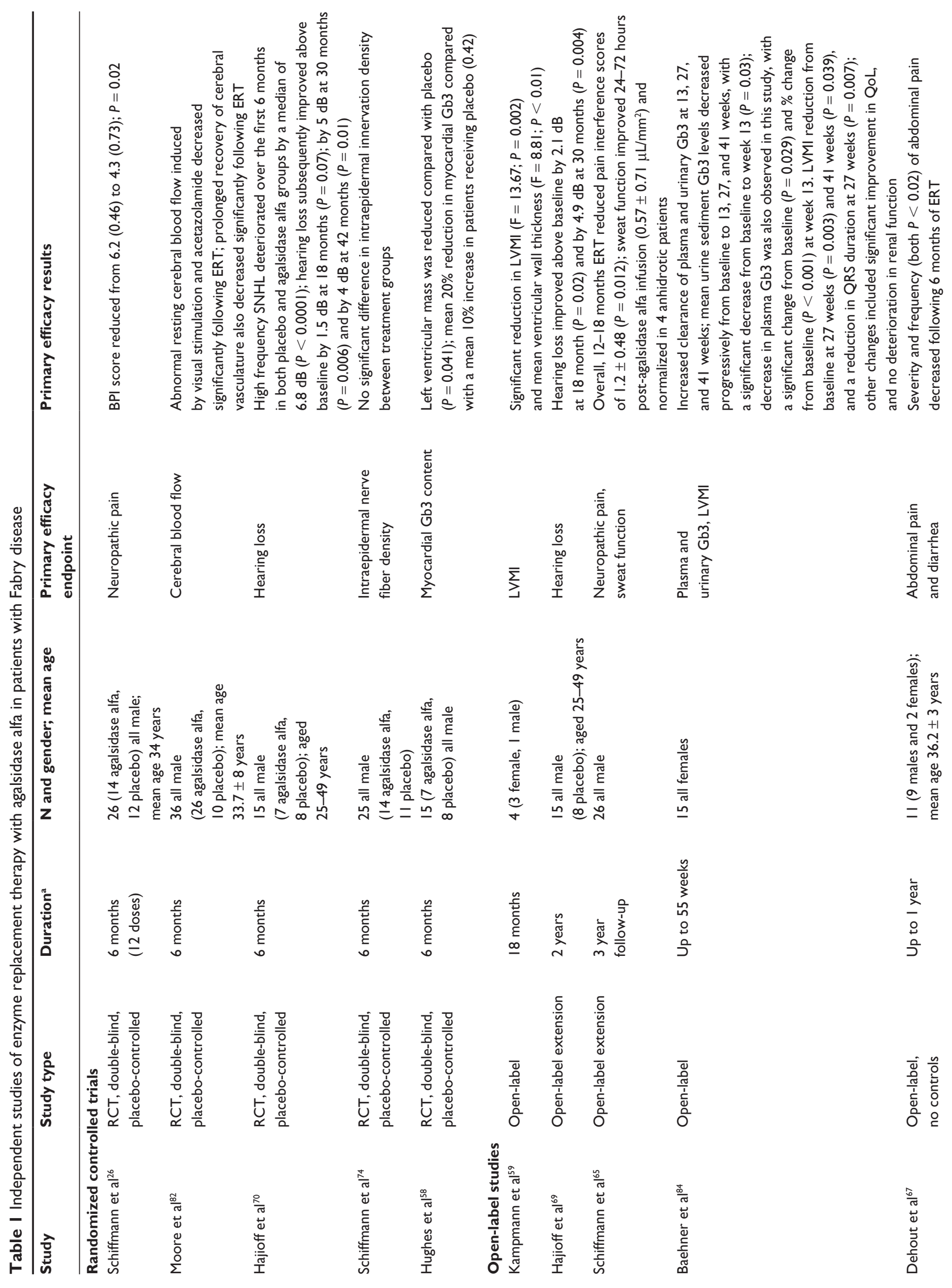



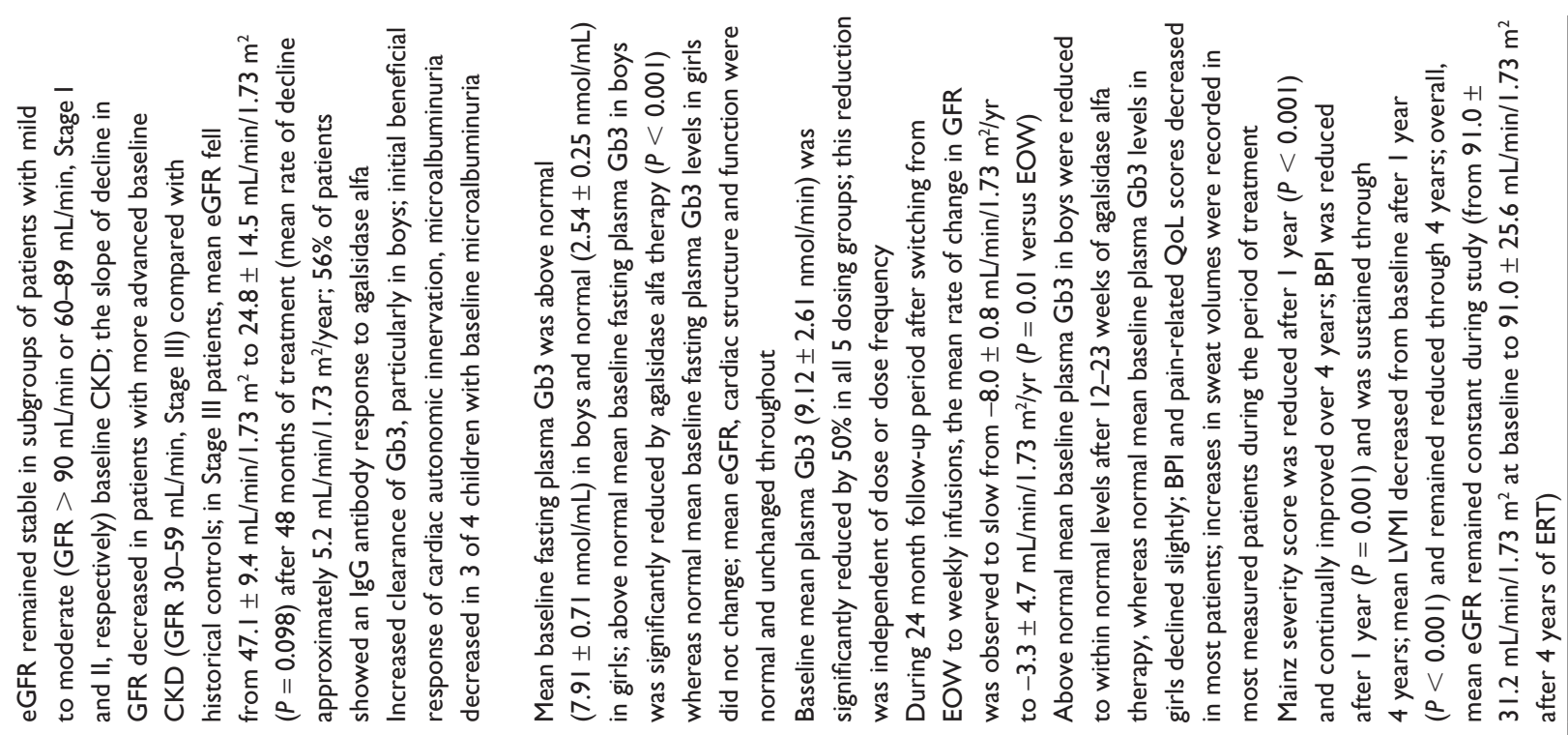

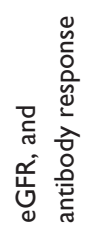
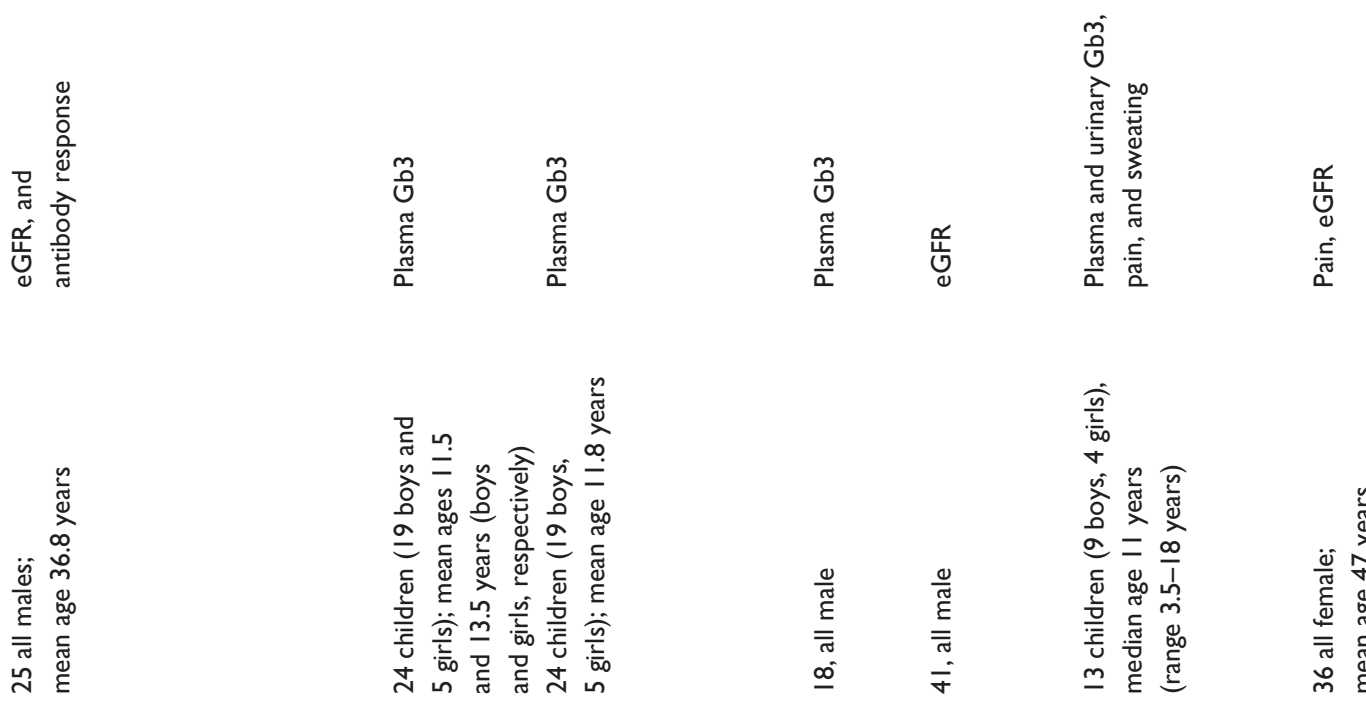

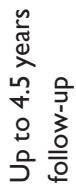
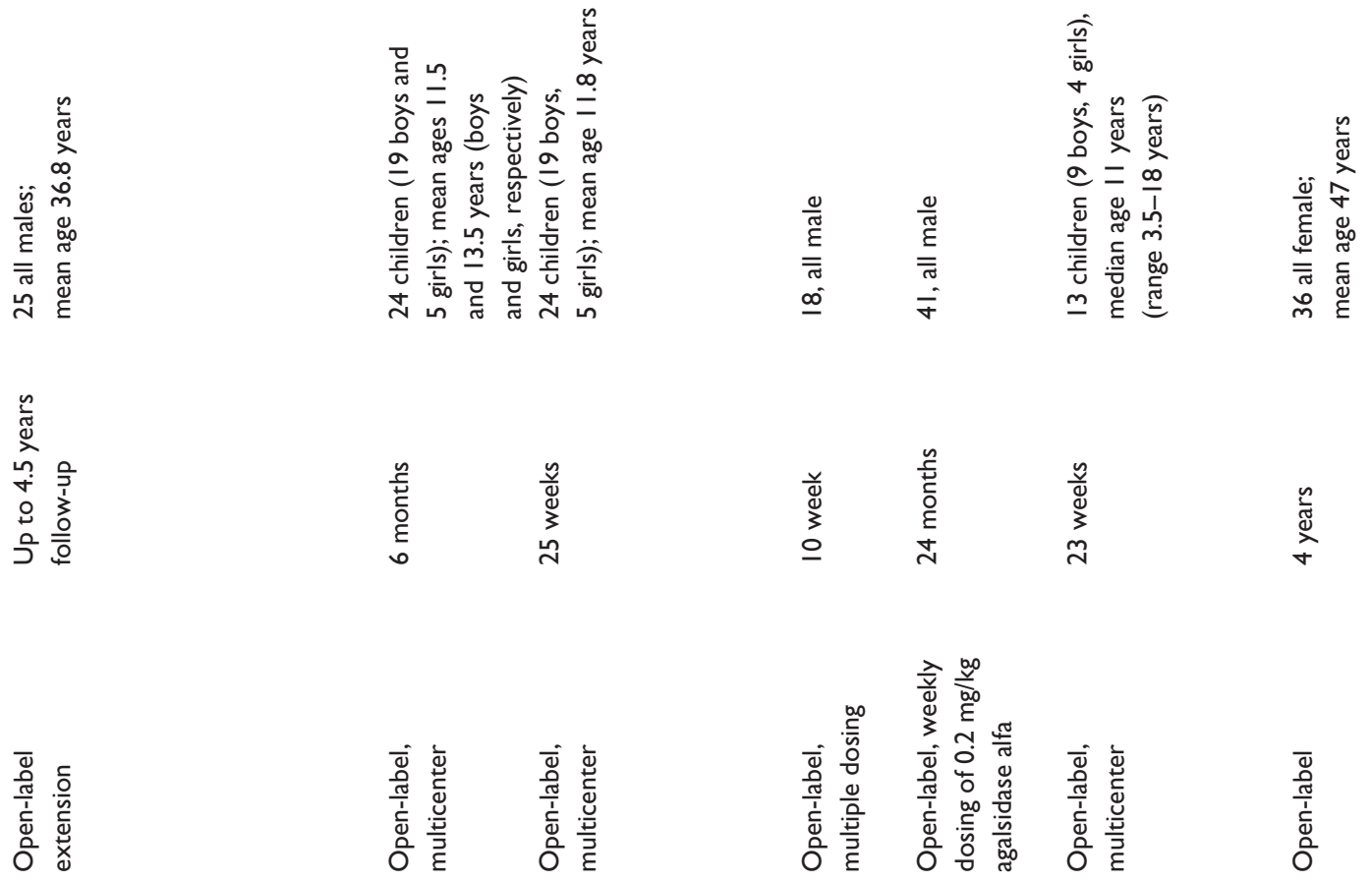

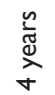

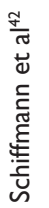

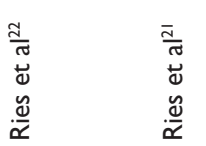

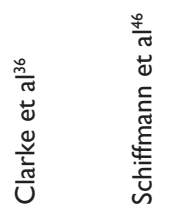

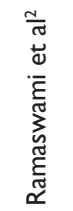

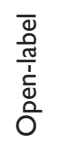




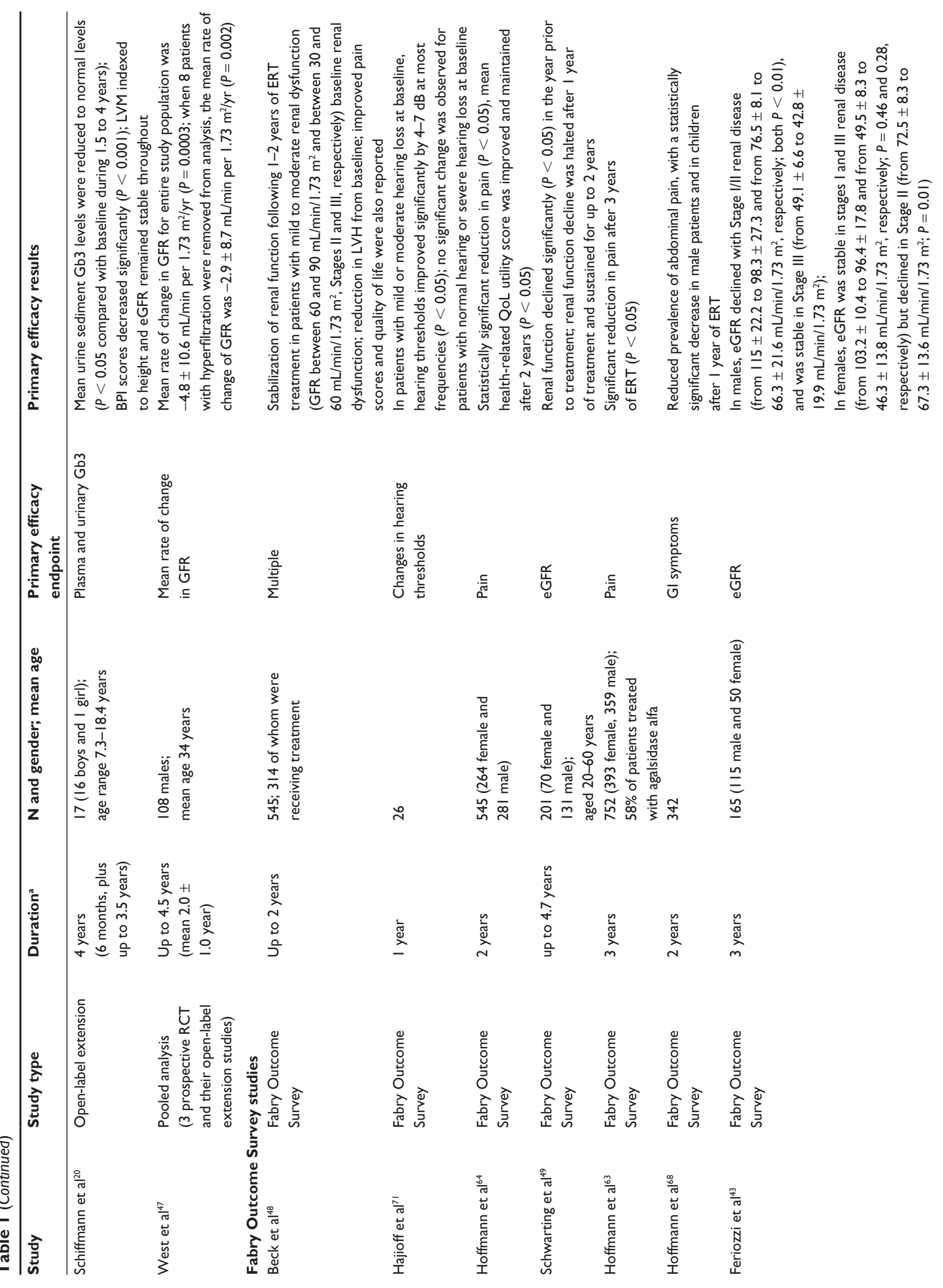




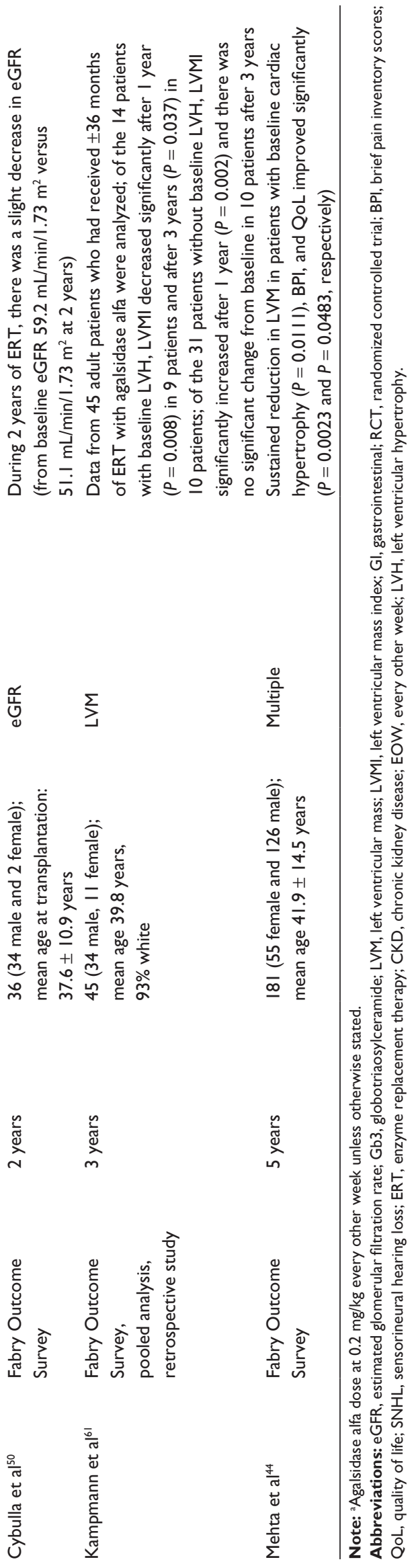

to $-3.3 \pm 4.7 \mathrm{~mL} / \mathrm{min} /$ year $(P=0.01$ versus every other week). Notably only two patients failed to improve their estimated glomerular filtration rate. ${ }^{46}$

In a pooled analysis of three randomized, placebocontrolled trials and their open-label extension studies involving male patients, the annual rate of decline in estimated glomerular filtration rate was $7.0 \mathrm{~mL} / \mathrm{min} / 1.73 \mathrm{~m}^{2}$ for 6 months of placebo treatment and $2.9 \mathrm{~mL} / \mathrm{min} / 1.73 \mathrm{~m}^{2}$ for 12-54 months of agalsidase alfa treatment, suggesting that agalsidase alfa treatment may slow the natural rate of glomerular filtration rate decline in male Fabry patients. ${ }^{47}$

Several studies from the analysis of patients in the Fabry Outcome Survey have reported that treatment with agalsidase alfa stabilizes estimated glomerular filtration rate in both male and female Fabry patients. ${ }^{43,44,48-50}$ Following 3 years of treatment, the overall change in estimated glomerular filtration rate for a large number of patients $(\mathrm{n}=165$, including 115 males and 50 females) receiving agalsidase alfa was $-1.68 \mathrm{~mL} / \mathrm{min} / 1.73 \mathrm{~m}^{2} /$ year for patients with baseline proteinuria $<0.50 \mathrm{~g} / 24$ hours $(\mathrm{n}=40)$ and $-3.98 \mathrm{~mL} / \mathrm{min} / 1.73 \mathrm{~m}^{2} /$ year for patients with baseline proteinuria $>0.50 \mathrm{~g} / 24$ hours $(\mathrm{n}=14) .{ }^{43}$ The most recent report from the Fabry Outcome Survey describes the observations in patients who have been treated with agalsidase alfa for 5 years. The mean annual decline in estimated glomerular filtration rate was $2.46 \mathrm{~mL} / \mathrm{min} / 1.73 \mathrm{~m}^{2}(\mathrm{n}=150$, males and females).

The efficacy of agalsidase beta replacement therapy on measures of kidney function was evaluated in two randomized controlled trials and in open-label studies. ${ }^{51-54}$ Creatinine clearance and estimated glomerular filtration rate remained stable after more than 3 years of treatment with agalsidase beta. ${ }^{51,54}$ Consistent with the results for agalsidase alfa, renal disease progression was observed for some patients receiving agalsidase beta who had more advanced disease at baseline. ${ }^{51,54}$

These results suggest that disease progression is related to disease severity in Fabry patients, thus early treatment could potentially provide greater benefit because there seems to be a point of no return, when the enzyme replacement therapy response is significantly reduced and renal failure is most probably irreversible. ${ }^{16}$

\section{Effects on cardiac manifestations}

Before the development of enzyme replacement therapy, cardiac involvement was the most common cause of premature mortality in Fabry disease ${ }^{55,56}$ Cardiac hypertrophy, arrhythmia, valvular insufficiency, and cardiac conduction 
abnormalities are common cardiac manifestations in Fabry disease. ${ }^{12,55,57}$ A small number of studies and observational data have provided evidence showing that $0.6-4.0$ years of treatment with agalsidase alfa results in a progressive decrease in interventricular septal thickness and a reduction or stabilization of left ventricular mass index. ${ }^{48,58-60}$

The effects of agalsidase alfa on cardiac structure and function were assessed in a randomized, double-blind, placebo-controlled study. In this study by Hughes et al, myocardial Gb3 content and reduction in left ventricular mass, as measured by magnetic resonance imaging, was assessed in 15 adult male Fabry patients following 6 months of enzyme replacement therapy with agalsidase alfa. Ten of the 15 patients enrolled had left ventricular hypertrophy at baseline, as measured by magnetic resonance imaging. Left ventricular mass increased in the placebo group by $21.8 \mathrm{~g}$ and decreased in the enzyme replacement therapy group by $11.5 \mathrm{~g}(P=0.041)$. This equated to a mean increase in left ventricular mass index for the placebo group of $12 \mathrm{~g} / \mathrm{m}^{2} \mathrm{com}-$ pared with a decrease in the treated group of $6.4 \mathrm{~g} / \mathrm{m}^{2}(P=0.02$ versus placebo).$^{58}$ Consistently, there was also a reduction in myocardial Gb3 content, albeit not statistically significant. ${ }^{58}$ These results were confirmed in the corresponding 2-year open-label extension study. In addition, left ventricular posterior and septal wall thickness was significantly decreased, suggesting remodeling had occurred. Throughout the 2-year extension trial, significant reductions in plasma Gb3 were maintained, reflecting increased metabolism of Gb3 deposits and a possible reduction in its pathogenic tissue storage. ${ }^{58}$ Echocardiographic data evaluation revealed a reduction in mean ventricular wall thickness $(P<0.05)$ and heightadjusted left ventricular mass (LVM/h) following 1-2 years for agalsidase alfa treatment in a large cohort of Fabry Outcome Survey patients with enlarged hearts at baseline. ${ }^{48}$ In a case study of four patients (three female heterozygotes and one male hemizygote), 18 months of agalsidase alfa treatment resulted in a significant continuous reduction in left ventricular mass indexed to body surface area $(\mathrm{F}=13.67$; $P=0.002)$ and mean ventricular wall thickness $(\mathrm{F}=8.81$; $P<0.01) .{ }^{59}$ More recently, Kampmann et al assessed the effectiveness of agalsidase alfa on LVM/h in male and female Fabry patients with and without baseline left ventricular hypertrophy. ${ }^{61}$ After 12 months of treatment in Fabry patients with left ventricular hypertrophy at baseline $(\mathrm{n}=14), \mathrm{LVM} / \mathrm{h}$ decreased significantly by $9.2 \pm 7.9 \mathrm{~g} / \mathrm{m}^{2.7}(\mathrm{n}=9 ; P=0.008)$. After 3 years of treatment in this subgroup, LVM/h decreased significantly by $5.1 \pm 7.5 \mathrm{~g} / \mathrm{m}^{2.7}(\mathrm{n}=10 ; P=0.037)$, suggesting that enzyme replacement therapy was associated with a significant reduction in LVM/h in patients with baseline left ventricular hypertrophy. ${ }^{61}$ After 12 months of treatment, in Fabry patients without baseline left ventricular hypertrophy $(\mathrm{n}=31), \mathrm{LVM} / \mathrm{h}$ increased significantly by $3.6 \pm 5.7 \mathrm{~g} / \mathrm{m}^{2.7}$ ( $\mathrm{n}=28 ; P=0.002)$. After 3 years of treatment in this subgroup, the increase in $\mathrm{LVM} / \mathrm{h}$ was not significantly different from baseline in 10 patients $\left(2.1 \pm 7.9 \mathrm{~g} / \mathrm{m}^{2.7}\right)$ suggesting stabilization of $\mathrm{LVM} / \mathrm{h}$ in patients without baseline left ventricular hypertrophy. Long-term (up to 5 years) treatment with agalsidase alfa in Fabry patients has been reported from the Fabry Outcome Survey observational database. ${ }^{44}$ In this population of males and females, stabilization or improvement in left ventricular mass index was seen in $71.9 \%$ (23/32) patients with baseline left ventricular hypertrophy, and in 92\% (23/25) patients without baseline left ventricular hypertrophy. ${ }^{44}$ In the group of 25 patients without baseline left ventricular hypertrophy, agalsidase alfa treatment resulted in small, statistically insignificant decreases in mean left ventricular mass index at years 1, 2 and 3, and a small, statistically insignificant increase in mean left ventricular mass index after 5 years. ${ }^{44}$ Notably, a significant improvement in ventricular function (midwall fractional shortening) was observed after years 1, 2, and 3 for patients with baseline left ventricular hypertrophy, and after 1 and 2 years in patients with normal baseline left ventricular mass index. Overall, midwall fractional shortening stabilized or improved in $62.1 \%$ (18/32) of subjects with baseline left ventricular hypertrophy, and in $95.2 \%(20 / 25)$ of subjects without baseline left ventricular hypertrophy. ${ }^{44}$

Interestingly, a recent study by Kampmann et al evaluated whether signs of cardiac manifestations were present in a population of 20 pediatric patients with Fabry disease (aged $\leq 18$ years). ${ }^{62}$ Mean baseline LVM/h was $45 \pm 2.3 \mathrm{~g} / \mathrm{m}^{2.7}$ and $47 \pm 3.4 \mathrm{~g} / \mathrm{m}^{2.7}$ in boys $(\mathrm{n}=8)$ and girls $(\mathrm{n}=12)$, respectively. All patients had LVM/ $\mathrm{h}^{2.7}$ greater than the 75 th percentile compared to healthy controls. ${ }^{62}$ Following a mean follow-up of 26 months, $85.7 \%(12 / 14)$ patients showed a mean increase of $7.5 \pm 3.2 \mathrm{~g} / \mathrm{m}^{2.7}$ in $\mathrm{LVM} / \mathrm{h}$. Analyses of heart rate variability revealed that male but not female Fabry patients had significantly reduced heart rate variability, suggesting a reduction in parasympathetic stimulation of the heart $(P<0.05){ }^{62}$ The authors of this study concluded that progressive cardiac involvement is common in pediatric Fabry patients. ${ }^{62}$

\section{Effects on pain and quality of life}

Acute neuropathic pain is a common early symptom of Fabry disease and occurs in about $80 \%$ of patients. ${ }^{37,63}$ Other neurological manifestations may include abnormal gut motility and pain, which have a negative impact on quality 
of life. ${ }^{63,64}$ Small fiber neuropathy is a cause of neuropathic pain. ${ }^{5,65}$ Acroparesthesia and hypohidrosis can also adversely affect quality of life. ${ }^{65,66}$

The effect of agalsidase alfa $0.2 \mathrm{mg} / \mathrm{kg}$ every other week on neuropathic pain was initially evaluated in a randomized, double-blind, placebo-controlled clinical trial of 26 adult males with Fabry disease. ${ }^{26}$ Following an initial six months of treatment with agalsidase alfa, mean ( \pm standard error) Brief Pain Inventory severity scores, reported as a primary endpoint, declined from $6.2 \pm 0.46$ to $4.3 \pm 0.73$, compared with no significant change in the placebo group $(P=0.02) .{ }^{26}$ Pain-related quality of life was also reduced for patients treated with agalsidase alfa versus placebo $(P=0.05) .^{26}$ Four of 11 patients treated with agalsidase alfa who were taking neuropathic pain medications at baseline discontinued these medications after 1-8 weeks compared with none of the patients $(n=11)$ taking neuropathic pain medications and receiving placebo $(P=0.03) .{ }^{26}$ Patients in the placebo group experienced a similar decline in Brief Pain Inventory severity scores 6 months after receiving agalsidase alfa treatment in the open-label extension study. ${ }^{65}$ No further reductions in pain scores were thereafter reported.

Several reports from the Fabry Outcome Survey indicate that agalsidase alfa has a beneficial effect on pain and quality of life. ${ }^{44,48,63,64}$ Recent reports from the Fabry Outcome Survey show that after 5 years of agalsidase alfa treatment, mean average pain was reduced from baseline. ${ }^{44}$ Reductions were significant at 2,3 , and 5 years $(P=0.0015, P=0.0128$, and $P=0.023$, respectively). Brief Pain Inventory severity scores also decreased, with significant reductions at 2 and 5 years $(P=0.0076$ and $P=0.0137$, respectively $){ }^{44}$ Clinically significant reductions (defined as improvement of $>1$ point on the Brief Pain Inventory) were observed for "average pain" ( $\mathrm{n}=32 ; 60.4 \%$ patients) and "worst pain" ( $\mathrm{n}=26 ; 53.1 \%$ patients). ${ }^{44}$ The EuroQoL questionnaire used to assess quality of life in the Fabry Outcome Survey revealed significant improvements compared with baseline following 1, 2, and 5 years of enzyme replacement therapy ( $P=0.0247, P=0.0026, P=0.0483$, respectively $)$, suggesting that agalsidase alfa may improve quality of life in both male and female Fabry patients. ${ }^{4}$

Agalsidase alfa has been reported to provide relief of gastrointestinal symptoms, such as abdominal pain and diarrhea. ${ }^{67,68}$ Dehout et al reported a decrease in frequency and severity of abdominal pain and diarrhea following 6 months of agalsidase alfa treatment. ${ }^{67}$ Following 1 year of agalsidase alfa treatment in both Fabry adults and children, the prevalence of abdominal pain, present in more than $50 \%$ of patients $(n=178)$ prior to enzyme replacement therapy, was significantly reduced in adults and children. ${ }^{68}$

\section{Effects on other organs/systems}

\section{Ear, nose and throat}

Progressive hearing impairment is a common manifestation in Fabry disease. ${ }^{69,70}$ Agalsidase alfa treatment for 6 months compared with placebo was reported to reverse hearing deterioration for 15 male Fabry patients in a randomized, double-blind study. ${ }^{70}$ In an open-label extension of this study, hearing loss slightly improved above baseline by $5 \mathrm{~dB}$ after 30 months of agalsidase alfa treatment $(P=0.004) .{ }^{69}$

Similarly, Fabry Outcome Survey data $(n=26)$ show improvements of similar magnitude ( 1 and $7 \mathrm{~dB}$ ) following agalsidase alfa treatment over 12 months in Fabry patients with moderately abnormal hearing thresholds at baseline. ${ }^{71}$ Hajioff et al conclude that agalsidase alfa treatment can stabilize and possibly improve hearing for Fabry patients who have not progressed to severe hearing loss. ${ }^{71}$ Clinical evidence is lacking to support any definitive conclusions that enzyme replacement therapy has any effect on hearing loss.

\section{Central nervous system}

Central nervous system manifestations of Fabry disease consist of cerebrovascular disease, including large vessel ectasia, large-vessel occlusive disease, and small vessel occlusive disease. ${ }^{72}$

A 3-year, open-label extension study reported that agalsidase alfa significantly improved thermal sensation, especially in the foot, as well as improved sweat function. ${ }^{65}$ A significant improvement in sympathetic skin responses in seven male patients during 2 years of agalsidase alfa has also been reported. ${ }^{73}$ Nerve fiber regeneration has not yet been observed..$^{74}$

Data from the Fabry Outcome Survey have demonstrated that cerebrovascular disease often coexists with advanced renal or cardiac disease ${ }^{75} \mathrm{~A}$ recent prospective study of cryptogenic stroke in young adults $(\mathrm{n}=721)$, using an $\alpha$-gal A enzymescreening assay, suggested that up to $1.2 \%$ of patients with stroke aged between $18-55$ years might have Fabry disease. ${ }^{76}$ However, the prevalence of unrecognized Fabry disease among young patients with ischemic strokes is unknown. ${ }^{76-78}$ The significant prevalence rate discrepancies reported in these studies (first ischemic stroke, prevalence range 0-2.17) is most probably due to differences in study populations, as outlined in the Wozniak et al study. ${ }^{77}$ Atypical variants of Fabry disease with late-onset cerebrovascular disease have been observed in a Belgian cohort of young patients with 
cerebrovascular disease, but the clinical relevance of this remains unclear at present. ${ }^{79}$ Cerebrovascular complications of stroke and transient ischemic attacks have been reported in $5 \%-27 \%$ of heterozygous Fabry females. ${ }^{5,37,80}$ One study has shown a higher prevalence of ischemic stroke and transient ischemic attacks in female Fabry patients compared with men ( $27 \%$ versus $12 \%$ for females and males, respectively). ${ }^{37}$

Changes in cerebral blood flow velocities have been published for Fabry patients treated with agalsidase alfa. ${ }^{72,81,82}$ Moore et al reported a significant decrease in cerebral hypoperfusion versus placebo following 6 months of treatment with agalsidase alfa in a randomized controlled trial of 26 male Fabry patients. ${ }^{72,82}$ No impact on the incidence of stroke was reported. ${ }^{72,82}$ In the open-label extension study, Moore et al demonstrated a reduction in elevated cerebral blood flow velocities after 18 months of agalsidase alfa therapy. ${ }^{81}$ The relationship between increased risk of stroke in Fabry disease and elevated regional cerebral blood flow remains unclear. ${ }^{72}$

\section{Effects in women}

In contrast with the long-held assumption that women with Fabry disease are rarely symptomatic, the majority of females who are heterozygous for disease causing mutations in the $\alpha$-gal A gene report clinical features of Fabry disease. .,6,83 $^{5}$ In one study, severe manifestations of disease were reported. For example, $77 \%$ of women reported neurological involvement, 59\% cardiac involvement, and 40\% renal involvement. ${ }^{83}$ Results from the Fabry Outcome Survey database and the baseline demographic data within it have been published recently, demonstrating that enzyme replacement therapy should not be restricted to hemizygous men, but should also be considered for heterozygous females and children. ${ }^{37}$ In 165 patients with Fabry neuropathy (including 50 female patients), Feriozzi et al demonstrated that enzyme replacement therapy with agalsidase alfa for 3 years might slow the decline in renal function in both male and females. ${ }^{43}$ In women, the mean estimated glomerular filtration rate decline was $1.20 \pm 3.28 \mathrm{~mL} / \mathrm{min} / 1.73 \mathrm{~m}^{2} /$ year, with a mean reduction in estimated glomerular filtration rate over 3 years of $5.0 \mathrm{~mL} / \mathrm{min} / 1.73 \mathrm{~m}^{2}(P<0.001){ }^{43}$

A single-center, open-label study of agalsidase alfa performed in 15 adult women with Fabry disease. In this clinical study by Baehner et al, female patients received enzyme replacement therapy for up to 55 weeks. Overall, agalsidase alfa in heterozygous females was demonstrated to regress left ventricular hypertrophy, improve quality of life, stabilize renal function, and reduce blood and urine glycolipid levels. ${ }^{84}$ Similarly, this study confirmed previous reports in male Fabry patients pertaining to the safety and pharmacokinetics of agalsidase alfa ${ }^{84} \mathrm{~A}$ significant decrease in left ventricular mass index from baseline was observed at 27 weeks $(P=0.003)$ and 41 weeks $(P=0.039)$, and a significant reduction in QRS interval was observed at week 27 $(P=0.007) .{ }^{84}$ Significant improvements in physical and general health subscales and the physical component score of the Short Form-36 questionnaire were also reported after 6 months of agalsidase alfa treatment. ${ }^{84}$ Evaluation of these 15 women continued in a 4-year, open-label extension study to evaluate the long-term response of females with Fabry disease to agalsidase alfa treatment. A total of 40 female patients were evaluated. Overall, in this long-term evaluation of the effects of enzyme replacement therapy on kidney function in women with Fabry disease, agalsidase alfa appeared to stabilize or improve estimated glomerular filtration rate in this cohort. ${ }^{6}$ Patients with Stage I and III chronic kidney disease remained mostly stable during the treatment period, only one of nine Stage I chronic kidney disease patients progressed to Stage II chronic kidney disease, and none of the Stage III chronic kidney disease patients $(n=3)$ showed disease progression. ${ }^{6}$ The subgroup of patients $(n=20)$ with moderately reduced kidney function at baseline (Stage II) showed a significant increase in estimated glomerular filtration rate after 1 year of treatment, which remained improved during 4 years of treatment. ${ }^{6}$ Six women who initiated angiotensin-converting enzyme inhibitors or angiotensin receptor blockers during the study demonstrated stable estimated glomerular filtration rate thereafter. ${ }^{6}$

Proteinuria has been reported in approximately $41 \%$ of women enrolled in the Fabry Outcome Survey, compared with $54 \%$ of men, although women with Fabry disease rarely develop end-stage renal failure. ${ }^{48}$ Following 4 years of treatment with agalsidase alfa, proteinuria was significantly reduced to a mean of $339 \pm 230 \mathrm{mg} / 24$ hours in 11 patients (35\%) with proteinuria $\geq 300 \mathrm{mg} / 24$ hours at baseline (mean $858 \pm 751 \mathrm{mg} / 24$ hours, $P<0.01) .{ }^{6}$ Brief Pain Inventory severity scores were reduced from $4.6 \pm 2.9$ at baseline to $3.3 \pm 2.9$ after 12 months $(P=0.001)$ and remained significantly reduced during the 4-year study. ${ }^{6}$ Left ventricular hypertrophy (defined as left ventricular mass $\geq 48 \mathrm{~g} / \mathrm{m}^{2.7}$ ) was present in $69 \%$ of females (25 of 36 patients) at baseline. ${ }^{6}$ $\mathrm{LVM} / \mathrm{h}$ was significantly reduced after 12 months of agalsidase alfa treatment in these 25 females with baseline left ventricular hypertrophy. Of these 25 patients, $13(52 \%)$ experienced decreases in LVM/h $>20 \%$, seven (28\%) demonstrated decreases in LVM/h of $10 \%-20 \%$, and two $(8 \%)$ 
demonstrated decreases between 7.2 and 3.5\%. After 4 years of treatment, seven of 25 patients with baseline left ventricular hypertrophy were classified as having normal $\mathrm{LVM} / \mathrm{h} .{ }^{6}$ Only one of the subgroup of 11 females with normal baseline $\mathrm{LVM} / \mathrm{h}\left(47.7 \mathrm{~g} / \mathrm{m}^{2.7}\right)$ was reclassified as having left ventricular hypertrophy at study completion $\left(50.3 \mathrm{~g} / \mathrm{m}^{2.7}\right){ }^{6}$ Based on the results from the Fabry Outcome Survey, Whybra et al suggest that women with any signs or symptoms of Fabry disease should be considered for enzyme replacement therapy. ${ }^{6}$

\section{Effects in children}

Observations from pediatric studies are consistent with the safety and efficacy profiles reported for agalsidase alfa in adults.,20,22,115 Efficacy and safety results of an initial 23-week, multicenter, open-label Fabry Outcome Survey trial in nine boys and four girls have been reported. ${ }^{2}$ The median age at treatment initiation was 11 years, and agalsidase alfa $0.2 \mathrm{mg} / \mathrm{kg}$ was administered as a 40 -minute infusion every 2 weeks. Only one male patient developed IgG antibodies during treatment. ${ }^{2}$ In this study, agalsidase alfa was well tolerated, and infusion-associated reactions were mild and resolved without sequelae. ${ }^{2}$ At baseline, all boys had increased plasma Gb3 levels, whereas plasma Gb3 concentrations were all within the normal range for girls. ${ }^{2}$ After 12 and 23 weeks of treatment, plasma Gb3 levels decreased to near normal levels in boys and declined slightly in girls. For all boys, the mean urinary $\mathrm{Gb3}$ :sphingomyelin ratio decreased from 4.6 at baseline to 2.5 after 23 weeks of treatment. ${ }^{2}$ In girls, the mean urinary Gb3:sphingomyelin ratio decreased from 0.7 at baseline to 0.3 at week $23 .^{2}$ The study also demonstrated reduced Brief Pain Inventory scores for "pain at its worst" from a mean baseline score of 2.8 to 1.5 at week 23 , and "pain on average" from a mean baseline score of 2.2 to 0.9 at week 23. ${ }^{2}$ Pain-related quality of life scores, including interference of pain on general activity, mood, walking ability, normal work, relationships, and enjoyment of life also decreased, and eight patients reported no pain interference in any quality of life categories at study end. ${ }^{2}$ Increased sweat volumes were recorded after 23 weeks of treatment with agalsidase alfa. ${ }^{2}$ Six of 10 patients who experienced acroparesthesia at baseline reported an improvement after 23 weeks of agalsidase alfa treatment. ${ }^{2}$

Similar clinical benefits have been documented in a second open-label study, which assessed the efficacy and safety of agalsidase alfa treatment for six months in 24 children with a mean age of 11.8 years. ${ }^{22}$ Overall, renal function remained normal following six months of enzyme replacement therapy (mean baseline estimated glomerular filtration rate $=121 \pm 5.0 \mathrm{~mL} / \mathrm{min} / 1.73 \mathrm{~m}^{2}$ and mean estimated glomerular filtration rate after 26 weeks $\left.=116 \pm 3.9 \mathrm{~mL} / \mathrm{min} / 1.73 \mathrm{~m}^{2}\right) .{ }^{22}$ Mean plasma Gb3 was significantly reduced among the 19 boys with elevated baseline fasting plasma $\mathrm{Gb} 3(P<0.001){ }^{22}$ In contrast, plasma Gb3 levels did not change among the five girls with normal baseline plasma Gb3 concentrations. ${ }^{22}$ Cardiac structure and function were normal and did not change during the study. ${ }^{22}$ In both boys and girls, mean LVM/h showed a nonsignificant decrease after 25 weeks of treatment. ${ }^{22}$ Of note, three children with high-normal LVM/h at baseline ( $>40 \mathrm{~g} / \mathrm{m}^{2.7}$, two girls and one boy) demonstrated a $15 \%$ mean decrease in LVM/h after 25 weeks of enzyme replacement therapy. ${ }^{22}$ Heart rate variability, which was decreased in boys compared with girls at baseline, improved significantly in boys. ${ }^{22}$ In addition, $55 \%$ of patients who were receiving anticonvulsive medication for neuropathic pain were able to decrease or cease their consumption. ${ }^{22}$

Long-term extension data of the latter study have been recently published. ${ }^{20}$ Schiffmann et al demonstrated that agalsidase alfa treatment for 4 years was well tolerated in children. ${ }^{20}$ Twenty-four children (19 boys and five girls), 10 of whom were treated for 4 years, showed significant reductions in pain scores, as well as sustained and improved heart rate variability in boys, estimated glomerular filtration rate, and LVM/h, which remained stable throughout the 4-year study period. ${ }^{20}$ Overall, $>93 \%$ of adverse events were reported as mild or moderate in severity for the 24 patients, and $<10 \%$ were deemed by the study investigators to be possibly related $(6.5 \%)$ or probably related $(3.4 \%)$ to study treatment. ${ }^{20}$ Only one male patient tested positive for IgG antibodies during this study. ${ }^{20}$

Ramaswami et al have recently evaluated the safety of enzyme replacement therapy with agalsidase alfa in young children enrolled in the Fabry Outcome Survey. ${ }^{85}$ This retrospective chart review identified eight children (mean age $5.0 \pm 1.6$ years) in the Fabry Outcome Survey who began treatment with agalsidase alfa $0.2 \mathrm{mg} / \mathrm{kg}$ intravenously every other week when younger than 7 years. Vital signs and adverse events were monitored throughout the study period. Glomerular filtration rate was estimated, and LVM/h (2.7) was assessed using echocardiography. Patients received 1.2-6.7 years of treatment (mean 4.2 years). Infusion reactions occurred in three patients and were of mild or moderate severity. IgG antibodies to agalsidase alfa were found in one patient who experienced two mild and 
one moderate infusion reactions. Mean glomerular filtration rate was within the normal range at baseline and remained normal. Left ventricular mass index was above the 75 th percentile for age-matched children in five of six patients evaluated at baseline. Only two patients exceeded this threshold at their last assessment. This study further emphasizes the importance of early diagnosis and follow-up of children with Fabry disease. ${ }^{85}$ The burden of Fabry disease in children is mostly due to neurological manifestations. ${ }^{34}$ Despite the small number of pediatric patient studies, Fabry Outcome Survey results to date reveal an overall improvement in pain, with either improvement or no change from baseline in most patients. $^{34}$ Infusion-associated reactions in pediatric Fabry Outcome Survey patients were generally mild and similar to those reported in adults. ${ }^{34}$

Early diagnosis is paramount, because renal pathology has been described even in young children (age range 7-18 years) who may have not yet developed overt proteinuria or decline in glomerular filtration rate; overt proteinuria has been reported in children as young as 10 years of age. ${ }^{86-88}$ Tøndel et al performed kidney biopsies in nine children, including two boys receiving enzyme replacement therapy. ${ }^{86}$ Notably, accumulation of Gb3 was found in nearly 7/9 (80\%) of the young patients as well as glomerular, interstitial, or vascular lesions, even prior to the development of clinical renal functional impairment. ${ }^{86}$ However, $7 / 9$ patients also had early onset microalbuminuria, thus emphasizing the importance of regular and early renal follow-up. ${ }^{86}$ Other pediatric studies have highlighted the importance of accurate diagnosis and careful monitoring for Fabry disease, because specific symptoms have been associated with greater disease severity and poorer prognosis. ${ }^{89,90}$ For example, retinal vascular tortuosity has been recognized as a predictive factor of a more severe illness in Fabry children. ${ }^{89,91}$ Allen et al investigated the correlation with Fabry genotype and systemic disease severity in children with ophthalmic manifestations. ${ }^{89}$ Overall, ophthalmic manifestations were found in $76 \%$ of children predicted to have complete loss-of-function $\alpha$-Gal A mutations $(P=0.003)$ and in only one patient with a genotype predicted to give some residual $\alpha$-Gal A function. ${ }^{89}$ Thirteen (50\%) of the 26 Fabry children were found to have corneal verticillata. ${ }^{89}$ Seven children $(27 \%)$ had evidence of retinal vascular tortuosity. ${ }^{89}$ All seven children (four girls and three boys) had more severe systemic disease suggestive of autonomic neuropathy, such as diarrhea and syncope. ${ }^{89}$ In another Fabry Outcome Survey study that investigated the epidemiology of hearing impairment and tinnitus in children with Fabry disease, Keilmann et al reported greater disease severity scores, measured by signs and symptoms questionnaires, in children with tinnitus..$^{90}$

\section{Comparator studies: agalsidase beta versus agalsidase alfa}

The clinical outcomes of agalsidase beta or agalsidase alfa in Fabry disease have been measured in a number of independent studies against a variety of endpoints. Most of these studies measured subclinical parameters rather than clinical outcomes per se. ${ }^{92}$ Both formulations are reported to provide several clinical benefits in the treatment of Fabry disease. However, comparison of therapeutic efficacy between the two distinct products is limited, probably due to the small number of randomized controlled trials, the small number of patients enrolled in these studies, different patient inclusion criteria, disease heterogeneity, short trial durations, as well as differences in outcome parameters and infused doses. ${ }^{28,35,93,94}$

To date, direct in vitro studies or studies in mice have shown no important differences between the two available enzyme preparations. ${ }^{24,25,28,35,95-98} \mathrm{~A}$ few recent head-to-head clinical studies of the relative efficacies of agalsidase beta compared with agalsidase alfa have been conducted. ${ }^{28,94,99,100}$ These trials are summarized in Table 2.

Vedder et al conducted an open-label prospective study comparing agalsidase alfa at its registered dose of $0.2 \mathrm{mg} / \mathrm{kg}$ every other week with an equivalent dose of agalsidase beta at $0.2 \mathrm{mg} / \mathrm{kg}$ every other week. ${ }^{28}$ The primary endpoint for this study was reduction in left ventricular mass after 1 and 2 years of enzyme replacement therapy. Twenty-six of the 29 patients enrolled had baseline left ventricular hypertrophy (13/14 subjects in the agalsidase alfa group and $13 / 15$ subjects in the agalsidase beta group). There was no difference in median cardiac mass between groups at baseline $(P=0.48)$. Overall, changes in left ventricular mass $(P=0.3)$ and other disease parameters did not differ between the two groups following 1-2 years of enzyme replacement therapy. A slightly increased decline in left ventricular mass observed in the agalsidase beta group (15\% versus $11 \%$ after 12 months and $11 \%$ versus $5 \%$ at 24 months, for beta versus alfa, respectively) was not deemed statistically significant by the study authors. ${ }^{28}$ Vedder et al also reported that $\alpha$-gal A antibodies in male adults occurred in approximately onethird of treated patients, but twice as often $(P=0.005)$ with agalsidase beta when dosed at $1.0 \mathrm{mg} / \mathrm{kg}$ every other week (8/10 patients) compared with agalsidase alfa at $0.2 \mathrm{mg} / \mathrm{kg}$ every other week (4/10 patients). This difference was not observed with agalsidase beta at the lower dose of $0.2 \mathrm{mg} / \mathrm{kg}$ every other week (6/10 patients).${ }^{99}$ 


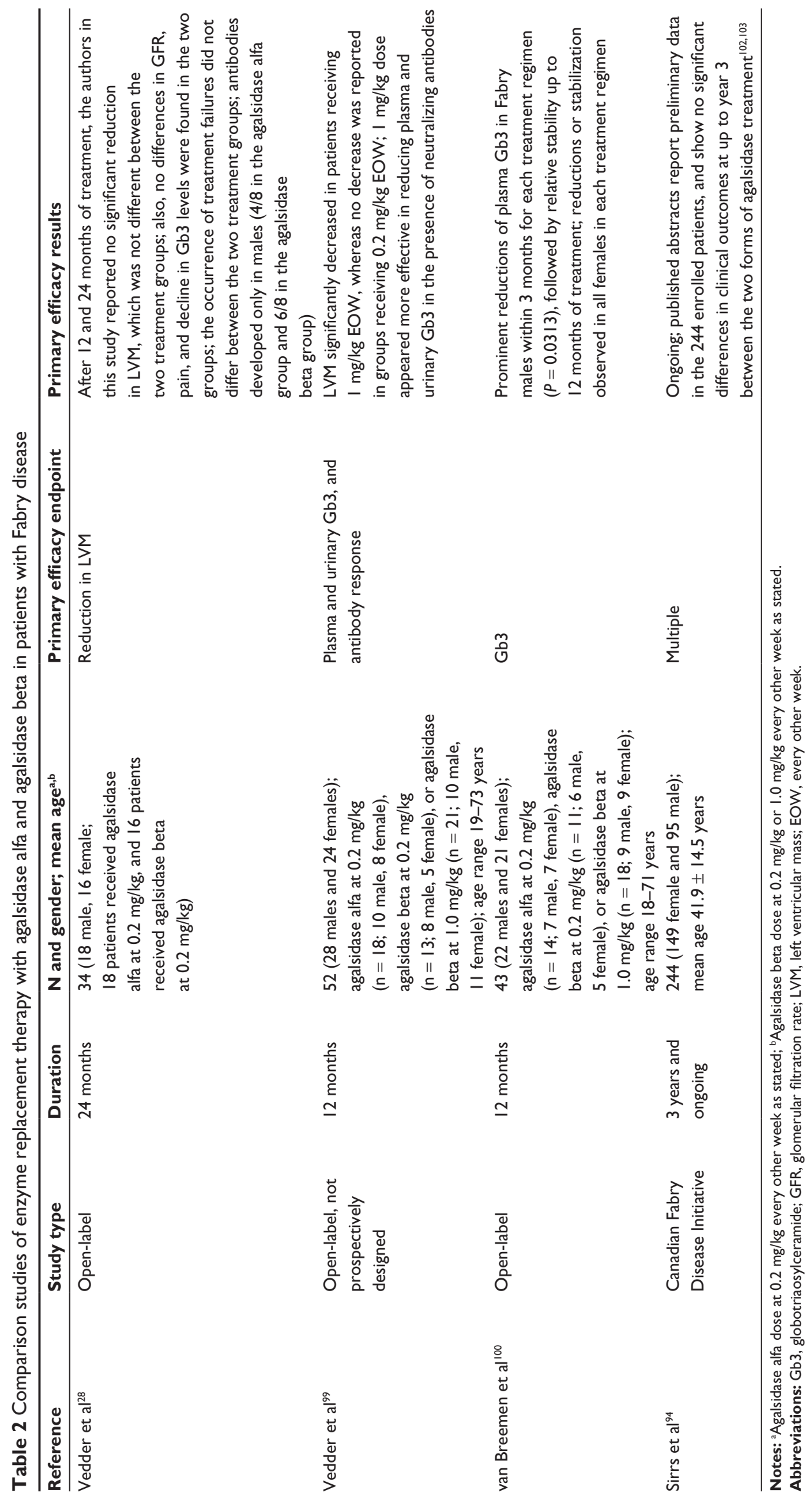


Consistent with other studies, none of the female patients developed antibodies. ${ }^{99}$ However, the impact of antibody formation on long-term clinical outcome remains unclear. ${ }^{99}$ A recent paper by Bénichou et al analyzed the potential impact of IgG antibodies to agalsidase beta on efficacy following 5 years of enzyme replacement therapy. ${ }^{101}$ No correlation was found between anti- $\alpha$-Gal A IgG titers and the onset of clinical events or the rate of change in estimated glomerular filtration rate. ${ }^{101} \mathrm{~A}$ statistically significant association was observed between anti- $\alpha$-Gal A IgG titers and Gb3 deposition in skin fibroblasts during treatment, suggesting impairment of $\mathrm{Gb} 3$ clearance in some patients with high antibody titers. ${ }^{101}$ Determination of the long-term impact of circulating anti- $\alpha$-Gal A antibodies on clinical outcomes requires continued monitoring during treatment. ${ }^{101}$

In a similar cohort of patients, Van Breeman et al recently described that agalsidase alfa $0.2 \mathrm{mg} / \mathrm{kg}$ every other week, agalsidase beta $0.2 \mathrm{mg} / \mathrm{kg}$ every other week, and agalsidase beta $1.0 \mathrm{mg} / \mathrm{kg}$ every other week resulted in marked decreases in elevated plasma Gb3 in Fabry males within three months $(P=0.0313)$, after which levels stabilized. ${ }^{100}$

The Canadian Fabry Disease Initiative is a large randomized controlled trial that has been initiated to provide robust multicenter national data on the natural history of the disease and on the comparative efficacy of agalsidase alfa and beta in enzyme replacement therapy-naïve Fabry patients. ${ }^{94}$ Multiple variables are to be studied during the 10 -year treatment investigation. Several published abstracts have reported preliminary data for the 244 enrolled patients, and showed no significant differences in clinical outcomes at up to year 3 between the two forms of agalsidase treatment. ${ }^{94,102,103}$ Future publication of this data will be particularly important to assess safety and clinical efficacy.

\section{Safety and tolerability}

The most common adverse events with agalsidase alfa are infusion-associated reactions, which occur in approximately $13.7 \%$ of treated Fabry patients in clinical trials. ${ }^{16,29,48}$ These adverse events are typically mild to moderate in severity, and include rigors, fever, nausea and vomiting, headache, chest pain, flushing, pruritus, rhinitis, tremor, dyspnea, somnolence, and acroparesthesia. ${ }^{6,16,48}$ The Fabry Outcome Survey data, based on treatment of 314 patients, described serious adverse events in 27 male and 11 female subjects, which were deemed not related to agalsidase therapy by the treating physician. ${ }^{48}$ Most of these events were attributed to the natural progression of Fabry disease, and included strokes, transient ischemic attacks, arrhythmias, renal dysfunction, vertigo, and sudden deafness. ${ }^{48}$ Combined analysis of longterm clinical trials shows that IgG antibodies have been observed in approximately $24 \%$ of the male patients after 3-12 months of treatment with agalsidase alfa, but no $\operatorname{IgE}$ antibodies or anaphylactic reactions have been noted. ${ }^{29}$ Interestingly, $7 \%$ of treated patients demonstrate immunological tolerance based on the disappearance of $\mathrm{IgG}$ antibodies over time. ${ }^{34,104}$ Antibodies to agalsidase alfa have not been shown to be associated with any clinically significant effects on safety or efficacy. ${ }^{29}$ Consistent with other studies, a long-term study of the efficacy and tolerability of agalsidase alfa in 36 female Fabry patients detected no antibodies during the treatment period. ${ }^{6}$ In vitro studies demonstrate that IgG antibodies cross-react similarly with both agalsidase alfa and beta, resulting in neutralization of $\alpha$-Gal A activity. ${ }^{34,105}$ Safety and tolerability with long-term agalsidase alfa treatment has been demonstrated, and no additional safety concerns have been raised in children or females. No safety concerns have been described for agalsidase alfa infusions used in the home setting. ${ }^{2,42,44}$

\section{Outstanding management issues}

The overall goal of enzyme replacement therapy is to prevent disease in younger patients, and to potentially halt disease progression and reverse underlying pathologic abnormalities in patients with more advanced disease. ${ }^{106-108}$ Results from clinical trials to date have highlighted that early initiation of enzyme replacement therapy may have the potential to delay and reverse the underlying clinical outcomes, such as renal failure, in patients with Fabry disease. ${ }^{18,42}$ Moreover, the use of enzyme replacement therapy in children is well tolerated, decreases pain, and improves pain-related quality of life. ${ }^{2,20,22}$ However, long-term follow-up is necessary to ascertain whether starting treatment in childhood will prevent and/or stabilize end organ damage and increase life expectancy. ${ }^{35}$

The possibility to halt or even reverse the progress of Fabry disease before irreversible damage organ occurs necessitates accurate and rapid diagnosis, monitoring, and treatment of the disease. ${ }^{89}$ Difficulties in comparing clearance of Gb3 and monitoring the clinical efficacy of enzyme replacement therapy in treated patients are highlighted by the wide variety of methodologies utilized in the studies to date. A reliable early biomarker or predictor of disease progression for both genders would prove valuable in this regard. ${ }^{35}$ The novel plasma biomarker, globotriaosylsphingosine (lyso-Gb3), is currently being studied as a potential biomarker for Fabry disease. ${ }^{109}$ Studies to date report increased plasma lyso-Gb3 in male patients, which was 
higher in cases of the classic phenotype, whereas plasma lyso-Gb3 was moderately increased in females, and this correlated with a decrease in $\alpha$-Gal A activity. ${ }^{109}$ Monitoring of microalbuminuria is currently recommended in Fabry patients, and could be readily used as a predictive indicator of early renal disease.$^{88}$ Early changes in renal morphology can occur in adults and children with normal glomerular filtration rate.$^{86,110}$ Precise assessment of glomerular filtration rate is therefore critical for early diagnosis, monitoring and treatment of nephropathy in these patients. ${ }^{88}$ Renal biopsy has been suggested as a potential tool to assess kidney disease severity accurately in pediatric Fabry patients, but further evaluation of this invasive procedure is warranted. ${ }^{86,88}$ Recent evaluation of different estimated glomerular filtration rate formulae, such as the Modification of Diet in Renal Disease method and the Schwartz formula commonly used in adult and pediatric Fabry patients, respectively, report overestimation of glomerular filtration rate, especially in the early stages of chronic kidney disease; it has been suggested that all Fabry Outcome Survey estimated glomerular filtration rate data in children be recalculated using the new abbreviated Schwartz measured glomerular filtration rate formula. ${ }^{88,111}$ The widely acknowledged lack of precision of estimated glomerular filtration rate measurements, particularly in pediatric patients, emphasizes the need for consensus guidelines and more accurate diagnostic tools. ${ }^{88,111}$

Consensus guidelines and recommendations for enzyme replacement therapy in Fabry patients have been published but, based on more recent data including Fabry Outcome Survey analyses, require updating. ${ }^{112-114}$ The European Medicines Agency have also issued treatment guidelines for patients with Fabry disease following continued shortages of agalsidase beta, which include adjusted dose regimens for adult male and female patients to $0.3 \mathrm{mg} / \mathrm{kg}$ every other week, switching to and starting treatment-naïve patients on alternative treatments, such as agalsidase alfa. ${ }^{115} \mathrm{~A}$ similar approach had been adopted for a shortage of Cerezyme ${ }^{\circledR}$ (imiglucerase for injection; Genzyme Corporation) for the treatment of Gaucher's disease. ${ }^{115}$ These extraordinary shortage circumstances have required a rapid global adaptation to current management strategies from industry, funding bodies, clinicians, patient organizations, and patients themselves. ${ }^{115}$ Due to the rarity of Fabry disease and the small numbers of patients available to be enrolled in large-scale, placebo-controlled clinical trials, data from registries, such as the Fabry Outcome Survey, are extremely valuable to further our understanding about the effect of enzyme replacement therapy in Fabry patients. Analysis of all randomized trials, open-label studies, and Fabry Outcome Survey data to date demonstrate clinical benefits of agalsidase alfa compared with placebo and provide further understanding of the natural history of Fabry disease, as well as the long-term safety of enzyme replacement therapy. Overall, agalsidase alfa treatment appears to be positively related to patients' health-related quality of life, ameliorating the natural course of the disease ${ }^{35}$ However, the value of enzyme replacement therapy for event-free survival, incidence and severity of comorbidities, as well as sustained improvement of patient health-related quality of life is not yet realized. ${ }^{35}$

Enzyme replacement therapy is a lifelong treatment for patients with Fabry disease, and home therapy has been proven to be beneficial for patients' quality of life whilst optimizing health care resources. The benefit of a 40-minute infusion and good safety profile of agalsidase alfa in adults and children has been regarded as more convenient in a home setting. ${ }^{42}$ Most patients in the UK have enzyme replacement therapy at home, and home care providers have experienced specialist nurses who provide this service, liaising closely with hospital clinicians and nurses. ${ }^{116}$ Recent studies have shown home infusion with agalsidase alfa to be convenient, and less stressful, as well as having a positive impact on patient satisfaction and possible cost reduction. ${ }^{42,116}$ The quality of evidence differs vastly between the studies mentioned in this review, from randomized controlled trials that are relatively short in duration, to open-label follow-up, and to registry data, which rely on good quality retrospective data, which has its own limitations. As a result, further randomized controlled studies may be required to define the appropriate time to initiate enzyme replacement therapy, as well as the optimal treatment dose for the individual patient. ${ }^{35}$ Careful and individualized treatment planning should be guided by accurate and earlier diagnosis of patients and timely assessment of organ damage. A study of two agalsidase beta dosing regimens in treatment-naïve male pediatric patients without severe symptoms will examine the efficacy and safety of two lower-dose regimens of Fabrazyme in male patients aged 5-18 years over the course of 5 years. Data from this study, described as Fabrazyme: Intervening Early at a Lower Dose (FIELD), may provide further supporting evidence for early and more flexible treatment, especially in pediatric patients and/or those with milder symptoms for Fabry disease. ${ }^{117}$

Preliminary data from the Canadian Fabry Disease Initiative suggest no significant differences in efficacy or safety between the two enzyme replacement therapy preparations. Owing to the recent production difficulties with agalsidase 
beta, further evaluation of a balanced and factual comparison of these two drugs may be hindered. ${ }^{115}$ In addition, there is a lack of in vitro studies available to allow relevant comparisons.

The systemic nature of Fabry disease necessitates a multidisciplinary management approach involving, for example, cardiologists, nephrologists, pediatricians, neurologists, ear, nose and throat specialists, psychologists, and specialist nurses. Other needs of Fabry patients and their families, such as genetic counseling and family screening, and treatment options such as home-based therapy should also be made available, because these are likely to have a positive impact on quality of life. ${ }^{34}$ In addition to enzyme replacement therapy, adjunctive therapies should be integrated, such as the use of appropriate analgesia for neuropathic pain, antihypertensives and the use of angiotensin-converting enzyme inhibitors and angiotensin receptor blockers for proteinuria, because these currently play an essential part in the optimal management of Fabry disease. ${ }^{16}$

\section{Conclusion}

Fabry disease is a slowly progressive disease with clinical manifestations often occurring in early childhood. Currently, in the absence of a suitable biomarker to establish the rate and severity of disease progression, monitoring patients regularly, both adults and children, for the onset of renal, cardiac, and central nervous system manifestations is essential to determine the timing of enzyme replacement therapy and adjunctive therapy. Over the past decade, the use of enzyme replacement therapy in patients with Fabry disease has significantly improved their quality of life and delays/ stabilizes disease progression.

Given the cost of enzyme replacement therapy and the burden of treatment over a prolonged period of time, it is vitally important to analyze long-term follow-up data to determine the point of no return with respect to disease progression, and thus optimizing the timing for commencing enzyme replacement therapy. Consensus guidelines of when to initiate enzyme replacement therapy are limited because of the rarity of the disease and a lack of reliable controlled studies designed to confirm the appropriate timing of initiation of enzyme replacement therapy. However, based on personal clinical experience, as well as recently published literature, the author's view is that initiation of enzyme replacement therapy must be considered carefully in a selected group of children with Fabry disease who have no residual enzyme activity and/or manifest early disease-related symptoms which significantly affect their quality of life.
These patient have a very high likelihood of developing significant complications related to Fabry disease.

Allen et al also correlated early ocular manifestations with genotype and overall disease severity in children with Fabry disease. ${ }^{89}$ Absent alfa-galactosidase A activity and genotype with loss of function mutations seem to be the best predictors of disease progression that we have in children currently, together with family history of a tendency to develop early strokes, and renal and cardiac complications. Once signs and symptoms develop, irreversible damage has probably already occurred. Regular follow-up will also enable clinicians to detect early evidence of cardiac, renal, or central nervous system disease in children, such as increase in left ventricular mass index above age-appropriate centiles, early onset of microalbuminuria, retinal vessel tortuosity, uncontrolled neuropathic pain, and recurrent fever pain crises. It is recommended that both male and female pediatric patients who remain asymptomatic in early childhood must have regular follow-up assessments at least once a year.

\section{Acknowledgements}

Dr Ramaswami has received travel and research grants from Shire Human Genetic Therapies and Genzyme Corporation. Klara Belzar, $\mathrm{PhD}$, is acknowledged for her assistance and was funded by Shire HGT. Dr Ramaswami was solely responsible for the contents of this manuscript.

\section{Disclosure}

The author reports no conflicts of interest in this work with the exception of that stated in the Acknowledgements.

\section{References}

1. Brady RO, Gal AE, Bradley RM, Martensson E, Warshaw AL, Laster L. Enzymatic defect in Fabry's disease. Ceramidetrihexosidase deficiency. N Engl J Med. 1967;276(21):1163-1167.

2. Ramaswami U, Wendt S, Pintos-Morell G, et al. Enzyme replacement therapy with agalsidase alfa in children with Fabry disease. Acta Paediatr. 2007;96(1):122-127.

3. MacDermot KD, Holmes A, Miners AH. Anderson-Fabry disease: Clinical manifestations and impact of disease in a cohort of 98 hemizygous males. J Med Genet. 2001;38(11):750-760.

4. Spada M, Pagliardini S, Yasuda M, et al. High incidence of later-onset Fabry disease revealed by newborn screening. Am J Hum Genet. 2006; 79(1):31-40.

5. MacDermot KD, Holmes A, Miners AH. Anderson-Fabry disease: Clinical manifestations and impact of disease in a cohort of 60 obligate carrier females. J Med Genet. 2001;38(11):769-775.

6. Whybra C, Miebach E, Mengel E, et al. A 4-year study of the efficacy and tolerability of enzyme replacement therapy with agalsidase alfa in 36 women with Fabry disease. Genet Med. 2009;11(6): 441-449.

7. Fabry J. Ein Beitrag zur Kenntniss der Purpura haemorrhagica nodularis (Purpura papulosa haemorrhagica Hebrae). Arch Dermatol Res. 1898;43(1):187-200. 
8. Anderson W. A case of angiokeratoma. Br J Dermatol. 1898;10:113.

9. Meikle PJ, Hopwood JJ, Clague AE, Carey WF. Prevalence of lysosomal storage disorders. JAMA. 1999;281(3):249-254.

10. Elleder M, Bradova V, Smid F, et al. Cardiocyte storage and hypertrophy as a sole manifestation of Fabry's disease. Report on a case simulating hypertrophic non-obstructive cardiomyopathy. Virchows Arch A Pathol Anat Histopathol. 1990;417(5):449-455.

11. von Scheidt W, Eng CM, Fitzmaurice TF, et al. An atypical variant of Fabry's disease with manifestations confined to the myocardium. N Engl J Med. 1991;324(6):395-399.

12. Nakao S, Takenaka T, Maeda M, et al. An atypical variant of Fabry's disease in men with left ventricular hypertrophy. $N$ Engl J Med. 1995; 333(5):288-293.

13. Nakao S, Kodama C, Takenaka T, et al. Fabry disease: Detection of undiagnosed hemodialysis patients and identification of a "renal variant" phenotype. Kidney Int. 2003;64(3):801-807.

14. Hwu WL, Chien YH, Lee NC, et al. Newborn screening for Fabry disease in Taiwan reveals a high incidence of the later-onset GLA mutation c. $936+919$ G $>$ A (IVS4+919G $>$ A). Hum Mutat. 2009; 30(10):1397-1405.

15. Hoffmann B. Fabry disease: Recent advances in pathology, diagnosis, treatment and monitoring. Orphanet J Rare Dis. 2009;4:21.

16. Mehta A, Beck M, Eyskens F, et al. Fabry disease: A review of current management strategies. QJM. 2010;103(9):641-659.

17. Parini R, Rigoldi M, Santus F, et al. Enzyme replacement therapy with agalsidase alfa in a cohort of Italian patients with Anderson-Fabry disease: Testing the effects with the Mainz Severity Score Index. Clin Genet. 2008;74(3):260-266.

18. Banikazemi M, Bultas J, Waldek S, et al. Agalsidase-beta therapy for advanced Fabry disease: A randomized trial. Ann Intern Med. 2007; 146(2):77-86.

19. Schiffmann R, Murray GJ, Treco D, et al. Infusion of alfagalactosidase A reduces tissue globotriaosylceramide storage in patients with Fabry disease. Proc Natl Acad Sci U S A. 2000;97(1): 365-370.

20. Schiffmann R, Martin RA, Reimschisel T, et al. Four-year prospective clinical trial of agalsidase alfa in children with Fabry disease. J Pediatr. 2010;156(5):832-837.

21. Ries M, Clarke JT, Whybra C, et al. Enzyme replacement in Fabry disease: Pharmacokinetics and pharmacodynamics of agalsidase alfa in children and adolescents. J Clin Pharmacol. 2007;47(10): 1222-1230.

22. Ries M, Clarke JT, Whybra C, et al. Enzyme-replacement therapy with agalsidase alfa in children with Fabry disease. Pediatrics. 2006; 118(3):924-932.

23. Blom D, Speijer D, Linthorst GE, Donker-Koopman WG, Strijland A, Aerts JM. Recombinant enzyme therapy for Fabry disease: Absence of editing of human alfa-galactosidase A mRNA. Am J Hum Genet. 2003;72(1):23-31.

24. Sakuraba H, Murata-Ohsawa M, Kawashima I, et al. Comparison of the effects of agalsidase alfa and agalsidase beta on cultured human Fabry fibroblasts and Fabry mice. J Hum Genet. 2006;51(3):180-188.

25. Lee K, Jin X, Zhang K, et al. A biochemical and pharmacological comparison of enzyme replacement therapies for the glycolipid storage disorder Fabry disease. Glycobiology. 2003;13(4):305-313.

26. Schiffmann R, Kopp JB, Austin HA 3rd, et al. Enzyme replacement therapy in Fabry disease: A randomized controlled trial. JAMA. 2001;285(21):2743-2749.

27. Eng CM, Guffon N, Wilcox WR, et al. Safety and efficacy of recombinant human alfa-galactosidase A - replacement therapy in Fabry's disease. N Engl J Med. 2001;345(1):9-16.

28. Vedder AC, Linthorst GE, Houge G, et al. Treatment of Fabry disease: Outcome of a comparative trial with agalsidase alfa or beta at a dose of $0.2 \mathrm{mg} / \mathrm{kg}$. PLoS One. 2007;2(7):e598.

29. Shire Human Genetic Therapies. Replagal: Summary of product characteristics. Danderyd, Sweden: Shire Human Genetic Therapies; 2010.
30. Genzyme Europe. Fabrazyme: Summary of product characteristics. Naarden, The Netherlands: Genzyme Europe; 2010.

31. Silversides A. Clinical trial for Fabry disease faces continuing hurdles. CMAJ. 2009;181(11):E251-E252.

32. European Medicines Agency. Supply shortage of Fabrazyme updated treatment recommendations required for adult male patients. 2009. Available from: www.ema.europa.eu/humandocs/PDFs/... fabrazyme/60258309en.pdf. Accessed January 1, 2011.

33. Mehta A. Agalsidase alfa for enzyme-replacement therapy in Fabry disease. Expert Rev Endocrinol Metab. 2010;5(5):641-652.

34. Mehta AB, Beck MP, Sunder-Plassmann G. Fabry Disease: Perspectives from 5 Years of FOS. Oxford: PharmaGenesis; 2006.

35. Pastores GM, Thadhani R. Enzyme-replacement therapy for AndersonFabry disease. Lancet. 2001;358(9282):601-603.

36. Clarke JT, West ML, Bultas J, Schiffmann R. The pharmacology of multiple regimens of agalsidase alfa enzyme replacement therapy for Fabry disease. Genet Med. 2007;9(8):504-509.

37. Mehta A, Ricci R, Widmer U, et al. Fabry disease defined: Baseline clinical manifestations of 366 patients in the Fabry Outcome Survey. Eur J Clin Invest. 2004;34(3):236-242.

38. Warnock DG, Daina E, Remuzzi G, West M. Enzyme replacement therapy and Fabry nephropathy. Clin J Am Soc Nephrol. 2009;5(2): 371-378.

39. Branton MH, Schiffmann R, Sabnis SG, et al. Natural history of Fabry renal disease: Influence of alfa-galactosidase $\mathrm{A}$ activity and genetic mutations on clinical course. Medicine (Baltimore). 2002;81(2):122-138.

40. Alroy J, Sabnis S, Kopp JB. Renal pathology in Fabry disease. J Am Soc Nephrol. 2002;13 Suppl 2:S134-S138.

41. Fogo AB, Bostad L, Svarstad E, et al. Scoring system for renal pathology in Fabry disease: Report of the International Study Group of Fabry Nephropathy (ISGFN). Nephrol Dial Transplant. 2009;25(7): 2168-2177.

42. Schiffmann R, Ries M, Timmons M, Flaherty JT, Brady RO. Longterm therapy with agalsidase alfa for Fabry disease: Safety and effects on renal function in a home infusion setting. Nephrol Dial Transplant. 2006;21(2):345-354.

43. Feriozzi S, Schwarting A, Sunder-Plassmann G, West M, Cybulla M. Agalsidase alfa slows the decline in renal function in patients with Fabry disease. Am J Nephrol. 2009;29(5):353-361.

44. Mehta A, Beck M, Elliott P, et al. Enzyme replacement therapy with agalsidase alfa in patients with Fabry's disease: An analysis of registry data. Lancet. 2009;374(9706):1986-1996.

45. Levey AS. Clinical practice. Nondiabetic kidney disease. NEngl J Med. 2002;347(19):1505-1511.

46. Schiffmann R, Askari H, Timmons M, et al. Weekly enzyme replacement therapy may slow decline of renal function in patients with Fabry disease who are on long-term biweekly dosing. J Am Soc Nephrol. 2007;18(5):1576-1583.

47. West M, Nicholls K, Mehta A, et al. Agalsidase alfa and kidney dysfunction in Fabry disease. J Am Soc Nephrol. 2009;20(5):1132-1139.

48. Beck M, Ricci R, Widmer U, et al. Fabry disease: Overall effects of agalsidase alfa treatment. Eur J Clin Invest. 2004;34(12):838-844.

49. Schwarting A, Dehout F, Feriozzi S, Beck M, Mehta A, SunderPlassmann G. Enzyme replacement therapy and renal function in 201 patients with Fabry disease. Clin Nephrol. 2006;66(2):77-84.

50. Cybulla M, Walter KN, Schwarting A, Divito R, Feriozzi S, SunderPlassmann G. Kidney transplantation in patients with Fabry disease. Transpl Int. 2009;22(4):475-481.

51. Wilcox WR, Banikazemi M, Guffon N, et al. Long-term safety and efficacy of enzyme replacement therapy for Fabry disease. Am J Hum Genet. 2004;75(1):65-74.

52. Eto Y, Ohashi T, Utsunomiya Y, et al. Enzyme replacement therapy in Japanese Fabry disease patients: The results of a phase 2 bridging study. J Inherit Metab Dis. 2005;28(4):575-583.

53. Breunig F, Weidemann F, Strotmann J, Knoll A, Wanner C. Clinical benefit of enzyme replacement therapy in Fabry disease. Kidney Int. 2006;69(7):1216-1221. 
54. Germain DP, Waldek S, Banikazemi M, et al. Sustained, long-term renal stabilization after 54 months of agalsidase beta therapy in patients with Fabry disease. J Am Soc Nephrol. 2007;18(5):1547-1557.

55. Brady RO, Schiffmann R. Clinical features of and recent advances in therapy for Fabry disease. JAMA. 2000;284(21):2771-2775.

56. Desnick RJ, Ioannou YA, Eng CM. A-galactosidase deficiency: Fabry disease. In: Scriver CR, Beaudet AL, Sly WS, Valle D, editors. The Metabolic and Molecular Basis of Inherited Disease. Vol 3. 8th ed. New York, NY: McGraw-Hill; 2001.

57. Chimenti C, Ricci R, Pieroni M, Natale L, Russo MA, Frustaci A. Cardiac variant of Fabry's disease mimicking hypertrophic cardiomyopathy. Cardiologia. 1999;44(5):469-473.

58. Hughes DA, Elliott PM, Shah J, et al. Effects of enzyme replacement therapy on the cardiomyopathy of Anderson-Fabry disease: A randomised, double-blind, placebo-controlled clinical trial of agalsidase alfa. Heart. 2008;94(2):153-158.

59. Kampmann C, Whybra C, Baehner F, Beck M. Enzyme replacement therapy in Anderson-Fabry cardiomyopathy. Heart Metab. 2002;18: 39-41.

60. Kampmann C. Enzyme replacement therapy and the heart. In: Mehta A, Beck M, Sunder-Plassmann G, editors. Fabry Disease: Perspectives from Five Years of FOS. Oxford: Oxford PharmaGenesis; 2006.

61. Kampmann C, Linhart A, Devereux RB, Schiffmann R. Effect of agalsidase alfa replacement therapy on Fabry disease-related hypertrophic cardiomyopathy: A 12- to 36-month, retrospective, blinded echocardiographic pooled analysis. Clin Ther. 2009;31(9): 1966-1976.

62. Kampmann C, Wiethoff CM, Whybra C, Baehner FA, Mengel E, Beck M. Cardiac manifestations of Anderson-Fabry disease in children and adolescents. Acta Paediatr. 2008;97(4):463-469.

63. Hoffmann B, Beck M, Sunder-Plassmann G, Borsini W, Ricci R, Mehta A. Nature and prevalence of pain in Fabry disease and its response to enzyme replacement therapy - a retrospective analysis from the Fabry Outcome Survey. Clin J Pain. 2007;23(6):535-542.

64. Hoffmann B, Garcia de Lorenzo A, Mehta A, Beck M, Widmer U, Ricci R. Effects of enzyme replacement therapy on pain and health related quality of life in patients with Fabry disease: Data from FOS (Fabry Outcome Survey). J Med Genet. 2005;42(3):247-252.

65. Schiffmann R, Floeter MK, Dambrosia JM, et al. Enzyme replacement therapy improves peripheral nerve and sweat function in Fabry disease. Muscle Nerve. 2003;28(6):703-710.

66. Gold KF, Pastores GM, Botteman MF, et al. Quality of life of patients with Fabry disease. Qual Life Res. 2002;11(4):317-327.

67. Dehout F, Roland D, Treille de Granseigne S, Guillaume B, Van Maldergem L. Relief of gastrointestinal symptoms under enzyme replacement therapy [corrected] in patients with Fabry disease. $J$ Inherit Metab Dis. 2004;27(4):499-505.

68. Hoffmann B, Schwarz M, Mehta A, Keshav S. Gastrointestinal symptoms in 342 patients with Fabry disease: Prevalence and response to enzyme replacement therapy. Clin Gastroenterol Hepatol. 2007;5(12):1447-1453.

69. Hajioff D, Enever Y, Quiney R, Zuckerman J, Mackermot K, Mehta A. Hearing loss in Fabry disease: The effect of agalsidase alfa replacement therapy. J Inherit Metab Dis. 2003;26(8):787-794.

70. Hajioff D, Goodwin S, Quiney R, Zuckerman J, MacDermot KD, Mehta A. Hearing improvement in patients with Fabry disease treated with agalsidase alfa. Acta Paediatr Suppl. 2003;92(443):28-30.

71. Hajioff D, Hegemann S, Conti G, et al. Agalsidase alfa and hearing in Fabry disease: Data from the Fabry Outcome Survey. Eur J Clin Invest. 2006;36(9):663-667.

72. Moore DF, Scott LT, Gladwin MT, et al. Regional cerebral hyperperfusion and nitric oxide pathway dysregulation in Fabry disease: Reversal by enzyme replacement therapy. Circulation. 2001; 104(13):1506-1512.

73. Jardim LB, Gomes I, Netto CB, et al. Improvement of sympathetic skin responses under enzyme replacement therapy in Fabry disease. $J$ Inherit Metab Dis. 2006;29(5):653-659.
74. Schiffmann R, Hauer P, Freeman B, et al. Enzyme replacement therapy and intraepidermal innervation density in Fabry disease. Muscle Nerve. 2006;34(1):53-56.

75. Mehta A, Ginsberg L. Natural history of the cerebrovascular complications of Fabry disease. Acta Paediatr Suppl. 2005;94(447):24-27.

76. Rolfs A, Bottcher T, Zschiesche M, et al. Prevalence of Fabry disease in patients with cryptogenic stroke: A prospective study. Lancet. 2005; 366(9499):1794-1796.

77. Wozniak MA, Kittner SJ, Tuhrim S, et al. Frequency of unrecognized Fabry disease among young European-American and African-American men with first ischemic stroke. Stroke. 2010;41(1):78-81.

78. Baptista MV, Ferreira S, Pinho EMT, et al. Mutations of the GLA gene in young patients with stroke: The PORTYSTROKE study screening genetic conditions in Portuguese young stroke patients. Stroke. 2010;41(3):431-436.

79. Brouns R, Thijs V, Eyskens F, et al. Belgian Fabry study: Prevalence of Fabry disease in a cohort of 1000 young patients with cerebrovascular disease. Stroke. 2010;41(5):863-868.

80. Galanos J, Nicholls K, Grigg L, Kiers L, Crawford A, Becker G. Clinical features of Fabry's disease in Australian patients. Intern Med J. 2002;32(12):575-584.

81. Moore DF, Altarescu G, Ling GS, et al. Elevated cerebral blood flow velocities in Fabry disease with reversal after enzyme replacement. Stroke. 2002;33(2):525-531.

82. Moore DF, Altarescu G, Herscovitch P, Schiffmann R. Enzyme replacement reverses abnormal cerebrovascular responses in Fabry disease. BMC Neurol. 2002;2:4

83. Deegan PB, Baehner AF, Barba Romero MA, Hughes DA, Kampmann C, Beck M. Natural history of Fabry disease in females in the Fabry Outcome Survey. J Med Genet. 2006;43(4):347-352.

84. Baehner F, Kampmann C, Whybra C, Miebach E, Wiethoff CM, Beck M. Enzyme replacement therapy in heterozygous females with Fabry disease: Results of a phase IIIB study. $J$ Inherit Metab Dis. 2003;26(7):617-627.

85. Ramaswami U, Parini R, Kampmann C, Beck M. Safety of agalsidase alfa in Fabry disease patients under 7 years. Acta Paediatr. November 29, 2010. [Epub ahead of print].

86. Tondel C, Bostad L, Hirth A, Svarstad E. Renal biopsy findings in children and adolescents with Fabry disease and minimal albuminuria. Am J Kidney Dis. 2008;51(5):767-776.

87. Sheth KJ, Roth DA, Adams MB. Early renal failure in Fabry's disease. Am J Kidney Dis. 1983;2(6):651-654.

88. Ramaswami U, Najafian B, Schieppati A, Mauer M, Bichet DG. Assessment of renal pathology and dysfunction in children with Fabry disease. Clin J Am Soc Nephrol. 2010;5(2):365-370.

89. Allen LE, Cosgrave EM, Kersey JP, Ramaswami U. Fabry disease in children: Correlation between ocular manifestations, genotype and systemic clinical severity. Br J Ophthalmol. 2010;94(12): 1602-1605.

90. Keilmann A, Hajioff D, Ramaswami U. Ear symptoms in children with Fabry disease: Data from the Fabry Outcome Survey. J Inherit Metab Dis. 2009;32(6):739-744.

91. Sodi A, Ioannidis AS, Mehta A, Davey C, Beck M, Pitz S. Ocular manifestations of Fabry's disease: Data from the Fabry Outcome Survey. Br J Ophthalmol. 2007;91(2):210-214.

92. Schaefer RM, Tylki-Szymanska A, Hilz MJ. Enzyme replacement therapy for Fabry disease: A systematic review of available evidence. Drugs. 2009;69(16):2179-2205.

93. Lidove O, Joly D, Barbey F, et al. Clinical results of enzyme replacement therapy in Fabry disease: A comprehensive review of literature. Int $J$ Clin Pract. 2007;61(2):293-302.

94. Sirrs S, Clarke JT, Bichet DG, et al. Baseline characteristics of patients enrolled in the Canadian Fabry Disease Initiative. Mol Genet Metab. 2009;99(4):367-373.

95. Bengtsson BA, Johansson JO, Hollak C, Linthorst G, FeldtRasmussen U. Enzyme replacement in Anderson-Fabry disease. Lancet. 2003;361(9354):352. 
96. Hopkin RJ, Bissler J, Grabowski GA. Comparative evaluation of alfagalactosidase A infusions for treatment of Fabry disease. Genet Med. 2003;5(3):144-153.

97. Desnick RJ. Enzyme replacement therapy for Fabry disease: Lessons from two alfa-galactosidase A orphan products and one FDA approval. Expert Opin Biol Ther. 2004;4(7):1167-1176.

98. Mignani R, Cagnoli L. Enzyme replacement therapy in Fabry's disease: Recent advances and clinical applications. J Nephrol. 2004; 17(3):354-363.

99. Vedder AC, Breunig F, Donker-Koopman WE, et al. Treatment of Fabry disease with different dosing regimens of agalsidase: Effects on antibody formation and GL-3. Mol Genet Metab. 2008;94(3):319-325.

100. van Breemen MJ, Rombach SM, Dekker N, et al. Reduction of elevated plasma globotriaosylsphingosine in patients with classic Fabry disease following enzyme replacement therapy. Biochim Biophys Acta. 2011; 1812(1):70-76.

101. Benichou B, Goyal S, Sung C, Norfleet AM, O'Brien F. A retrospective analysis of the potential impact of IgG antibodies to agalsidase beta on efficacy during enzyme replacement therapy for Fabry disease. Mol Genet Metab. 2009;96(1):4-12.

102. West MFG, LeMoine K, Bichet D, et al. The Canadian Fabry Disease Initiative: A randomised controlled trial of agalsidase therapy in Fabry neuropathy. Int J Clin Pharmacol Ther. 2010;48(Suppl 1):S51.

103. West MLK, Bichet D, Clarke J, et al. A randomised controlled trial of enzyme replacement therapy in Fabry disease: The Canadian disease initiative at year three. Mol Genet Metab. 2010;99(S39):Abstract 148.

104. European Agency for the Evaluation of Medicinal Products. Product Overview. Replagal. 2010. Available from: http://www. ema.europa.eu/ema/index.jsp?curl=pages/medicines/human/ medicines/000369/human_med_001029.jsp\&mid=WC0b01ac058001 d125\&murl=menus/medicines/medicines.jsp\&jsenabled=true. Accessed February 2, 2011.

105. Schellekens H. Bioequivalence and the immunogenicity of biopharmaceuticals. Nat Rev Drug Discov. 2002;1(6):457-462.

106. Rosenthal DI, Doppelt SH, Mankin HJ, et al. Enzyme replacement therapy for Gaucher disease: Skeletal responses to macrophagetargeted glucocerebrosidase. Pediatrics. 1995;96(4 Pt 1):629-637.
107. Grabowski GA, Leslie N, Wenstrup R. Enzyme therapy for Gaucher disease: The first 5 years. Blood Rev. 1998;12(2):115-133.

108. Mistry PK. Gaucher's disease: A model for modern management of a genetic disease. J Hepatol. 1999;30 Suppl 1:1-5.

109. Togawa T, Kodama T, Suzuki T, et al. Plasma globotriaosylsphingosine as a biomarker of Fabry disease. Mol Genet Metab. 2010;100(3): 257-261

110. Gubler MC, Lenoir G, Grunfeld JP, Ulmann A, Droz D, Habib R. Early renal changes in hemizygous and heterozygous patients with Fabry's disease. Kidney Int. 1978;13(3):223-235.

111. Tondel C, Ramaswami U, Aakre KM, Wijburg F, Bouwman M, Svarstad E. Monitoring renal function in children with Fabry disease: Comparisons of measured and creatinine-based estimated glomerular filtration rate. Nephrol Dial Transplant. 2009;25(5):1507-1513.

112. Desnick RJ, Brady R, Barranger J, et al. Fabry disease, an underrecognized multisystemic disorder: Expert recommendations for diagnosis, management, and enzyme replacement therapy. Ann Intern Med. 2003;138(4):338-346.

113. Eng CM, Germain DP, Banikazemi M, et al. Fabry disease: Guidelines for the evaluation and management of multi-organ system involvement. Genet Med. 2006;8(9):539-548.

114. Hughes DA, Ramaswami U, Elliott P, et al; National Specialist Commissioning Advisory Group (NSCAG). Guidelines for the Diagnosis and Management of Anderson-Fabry Disease. London, UK: National Commissioning Group of the National Health Service; 2005.

115. European Medicines Agency. Questions and answers on the shortages of Cerezyme and Fabrazyme. 2009. Available from: www.ema.europa eu/humandocs/PDFs/EPAR/Cerezyme/51076609en.pdf. Accessed February $02,2011$.

116. Milligan A, Hughes D, Goodwin S, Richfield L, Mehta A. Intravenous enzyme replacement therapy: Better in home or hospital? Br J Nurs. 2006;15(6):330-333.

117. ClinicalTrials.gov. A study of two Fabrazyme (agalsidase beta) dosing regimens in treatment-naïve, male pediatric patients without severe symptoms (FIELD). Available from: http://clinicaltrials.gov/ct2/show/ NCT00701415. Accessed February 02, 2011.

\section{Publish your work in this journal}

Drug Design, Development and Therapy is an international, peerreviewed open-access journal that spans the spectrum of drug design and development through to clinical applications. Clinical outcomes, patient safety, and programs for the development and effective, safe, and sustained use of medicines are a feature of the journal, which

\section{Dovepress}

has also been accepted for indexing on PubMed Central. The manuscript management system is completely online and includes a very quick and fair peer-review system, which is all easy to use. Visit http://www.dovepress.com/testimonials.php to read real quotes from published authors. 